\title{
Comparison of different models for EBCD calculation in the TJ-II Stellarator
}

\author{
J. M. García-Regaña ${ }^{1}$, F. Castejón ${ }^{1}$, A. Cappa ${ }^{1}$, \\ N. B. Marushchenko ${ }^{2}$ and M. Tereshchenko ${ }^{3}$ \\ ${ }^{1}$ Laboratorio Nacional de Fusión, EURATOM/CIEMAT, Madrid, Spain \\ 2 Max-Planck-Institut für Plasmaphysik, EURATOM-Association, Greifswald, \\ Germany \\ 3 Prokhorov Institute of General Physics, Moscow, Russia / BIFI: Instituto de \\ Biocomputación y Física de Sistemas Complejos, Zaragoza, Spain
}

E-mail: josemanuel.garcia@ciemat.es

\begin{abstract}
.
In the present work, we have compared different linear methods to estimate the Electron Bernstein Current Drive (EBCD). The expressions for the current drive efficiency have been plugged to the ray tracing code TRUBA, which was used in previous works for EBW heating studies in the TJ-II stellarator. This device is taken here as an example for this comparison.

The driven current is calculated for different densities and temperatures, as well as launching directions of the heating beam, which is a critical issue in the O-X-B mode conversion scenario considered in TJ-II. The range of applicability of each model is discussed. The influence of the Ohkawa, relativistic and frictional trapping effects on the total current generated is studied by comparing the results obtained by pairs of models that include and neglect those effects. The Ohkawa effect has resulted to be the less important. Although the relativistic effects are not negligible, the main disagreement between the results arises from including or not momentum conservation and neglecting frictional trapping effects. The total EBCD current drive efficiency calculated is in all cases greater than the experimental ECCD one, previously measured in TJ-II. The results presented in this work are the guideline for future experiments in this device.
\end{abstract}




\section{Introduction}

Current generation in plasmas by launching waves in the range of the electron cyclotron frequency has been widely studied theoretically and experimentally. This wave-induced current plays an important role in the plasma stability and confinement in stellarators, enabling deleterious plasma currents compensation, rotational transform profile shaping and magnetohydrodynamic instabilities control. It is also expected to contribute to overcome the pulsed operation in tokamaks. A description of the progress made in these directions, the physics of electron cyclotron current drive (ECCD) itself and a recent benchmarking of codes are reviewed in references $[1,2,3,4,5]$. On the other hand, the density cut-off of the electromagnetic modes of propagation prevents heating and current drive at high density regimes. For this reason, Electron Bernstein Waves (EBWs) [6] becomes necessary to attain non-ohmic heating of overdense plasmas and the subsequent current drive generation. EBWs electrostatic nature makes them achieve a parallel refractive index larger than unity. This feature expands the resonance condition domain and displaces it to further suprathermal values of parallel momentum, not so strongly affected by the proximity to the trapping cone. Thus, the deterioration of the current efficiency due to the intersection of the resonance curve and the trapping cone, or the counteracting Ohkawa contribution to the current, is expected not to be as strong as in the case of EC waves [7]. For EBWs as source for plasma heating, diagnostic, and current drive see Refs. [8, 9].

In the TJ-II stellarator [10], where electron density can reach up to $8.0 \times 10^{19} \mathrm{~m}^{-3}$, ECRH heating is limited by the second harmonic $(53.2 \mathrm{GHz}) \mathrm{X}$-mode cutoff density, $1.7 \times 10^{19} \mathrm{~m}^{-3}$. For this reason an EBWs heating system is necessary for electron heating above that density. Bernstein waves are expected to be excited through the O-X-B mode conversion process [13] at first harmonic (28 GHz) with up to $300 \mathrm{~kW}$ of available power. The lack of available injection ports and the need of low field side launching inherent to the $\mathrm{O}-\mathrm{X}-\mathrm{B}$ scenario requires the use of an internal mirror. Moreover, the optimum beam for maximum O-X conversion efficiency is such that its wavefront curvature, close to the LCFS, matches the plasma surface curvature and therefore and elliptical focusing mirror is needed. In order to achieve the experimental optimization of the O-X mode conversion, the mirror is also steerable around the optimum theoretical position and the changes in the focal length for different launching directions are expected to be of minor importance in the O-X mode conversion. For details on the design of the EBW heating system see e.g. Refs. [11] and [12].

This work aims at the estimation of the Electron Bernstein Current Drive (EBCD) expected in the TJ-II EBW-heated plasmas using different linear models for the current drive calculation. Since the different physics underlying each calculation model provides noticeable variations in the final results, a comparison between them is carried out. Although the calculation is performed using the TJ-II magnetic configuration, the conclusions regarding the usage of each model varying the electron density and temperature can be extrapolated to any other device where EBCD is being considered. 
The wave propagation and absorption properties used in the EBCD calculation have been obtained with the ray tracing code TRUBA [14].

The remainder of the paper is organized as follows: Section 2 will describe briefly the ray tracing code TRUBA, while section 3 deal with the current generation mechanisms and models considered; Section 4 will show results of current drive efficiency, where the subsection 4.1 will focus on the results for a single ray simulation, while subsections 4.2 and 4.3 are devoted to explore, using multi-ray simulations, the current generated under different plasma parameters and launching mirror positions respectively. Finally a summary, conclusion and future work are presented in Sect. 5. 


\section{The ray tracing code: TRUBA}

For a given frequency $\omega$, The TRUBA code solves the standard ray tracing equations

$$
\frac{d \mathbf{R}(\sigma)}{d \sigma}=-\left[\frac{\partial \mathcal{H}}{\partial \mathbf{N}}\right]_{\mathbf{R}(\sigma), \mathbf{N}(\sigma)} \frac{d \mathbf{N}(\sigma)}{d \sigma}=\left[\frac{\partial \mathcal{H}}{\partial \mathbf{R}}\right]_{\mathbf{R}(\sigma), \mathbf{N}(\sigma)},
$$

where $\mathbf{R}(\sigma)$ are the space coordinates of the ray, $\mathbf{N}(\sigma)$ is the normalized wave vector and $\sigma$ is the arc parameter of the ray. The ray Hamiltonian for a given wave mode, $\mathcal{H}(\mathbf{R}, \mathbf{N})$, is usually taken to be proportional to the real part of the mode-pertinent eigenvalue $\Lambda^{(m)}$ of the plane-wave dispersion tensor $\Lambda$. This allows expressing $\mathcal{H}(\mathbf{R}, \mathbf{N})$ as $[15]$

$$
\mathcal{H}=f \prod_{j=1,2,3} \operatorname{Re} \Lambda^{(j)}=f \operatorname{det}\left(\Lambda^{\mathrm{H}}\right)
$$

where $f=f(\mathbf{R}, \mathbf{N})$ is an arbitrary non-vanishing real function and $\boldsymbol{\Lambda}^{\mathrm{H}}$ is the Hermitian part of the dispersion tensor. To a first-order infinitesimal of inhomogeneities, the power transfer equation corresponding to the ray tracing equations (1) with the Hamiltonian (2) is

$$
\frac{d P}{d \sigma}=-2 \frac{\omega}{c} P \chi\left[f \operatorname{Im}\left(\operatorname{det}(\boldsymbol{\Lambda})-\operatorname{det}\left(\boldsymbol{\Lambda}^{\mathrm{A}}\right)\right)\right]_{\mathbf{R}(\sigma), \mathbf{N}(\sigma)} .
$$

Here, $P$ is the total wave power flux, proportional to $|\mathbf{E}|^{2}\left[\partial \operatorname{Re} \Lambda^{(m)} / \partial \mathbf{N}\right]_{\mathbf{R}(\sigma), \mathbf{N}(\sigma)}$, $\mathbf{E}$ is the local quasi-plane wave field, $\boldsymbol{\Lambda}^{\mathrm{A}}$ is the anti-Hermitian part of the dispersion tensor, and $\chi=\left[\operatorname{Im} \Lambda^{(m)}\right]_{\mathbf{R},(c / \omega) \nabla(\arg \mathbf{E})} /\left[\operatorname{Im} \Lambda^{(m)}\right]_{\mathbf{R}, \mathbf{N}}$. Within this approximation the value of $\chi$ is very close to unity.

The code gives the possibility to use a non-relativistic (see e.g. [16]) or a weakly relativistic [17] hot Maxwellian plasma dispersion tensor. This latter is valid for $N_{\perp}^{2} \ll\left(m_{e} c^{2} / T_{e}\right)\left(\omega_{c e} / \omega\right)^{2}$, and thus suitable for EBWs at low cyclotron harmonics. A comparison between the results obtained for the EBWs propagation and absorption in TJ-II considering both expressions of the dispersion tensor is discussed in ref. [12]. Since the goal of the present paper is the calculation and comparison of the current drive obtained using different models, the non-relativistic dispersion tensor has been used. Although it is not explicitly shown, the difference between the results provided by each current drive model is larger or similar to the difference obtained with and without considering the relativistic effects in the ray tracing code, that is in the calculation of $N_{\|}$and $P$.

In the computation of the $\mathrm{O}-\mathrm{X}-\mathrm{B} 1$ scenario, the ray tracing procedure is forced to reasonably describe the transmission of $\mathrm{O}$ wave power (if any) through the opacity region, whose boundaries in the cold-plasma limit are $q_{e}=1$ and $q_{e}=\left(1+\sqrt{u_{e}}\right)\left(1-N_{\|}^{2}\right)$, where $q_{e}=\left(\omega_{p e} / \omega\right)^{2}, u_{e}=\left(\omega_{c e} / \omega\right)^{2}$, and $\omega_{p e}, \omega_{c e}$ are the electron plasma and cyclotron frequencies. This tunnelling makes possible the subsequent $\mathrm{O}-\mathrm{X}$ mode conversion, provided that the plasma is dense enough to ensure the condition $q_{e}=$ 
$1+u_{e}\left[\left(1-N_{\|}^{2}\right) / 2 N_{\|}\right]^{2}$. The TRUBA code proceeds with the expected tunnelling applying the following technique. If the reflection point is revealed along the ray trajectory of the incident $\mathrm{O}$ wave, and the width of the opposed evanescent layer is small enough, the launching point of the transmitted ray is to be found by moving from the reflection point towards the direction of density gradient until the dispersion relation is fulfilled again. It was shown in [18] that the ray trajectory continued from this latter point is asymptotically equivalent to the limiting central trajectory of the transmitted part of the wave packet. The wave vector of the launching ray has to be equated with its value at the reflection point. The transmission efficiency $\eta$ (i.e. the fraction of the power which is transmitted) is calculated using the one-dimensional O mode tunnelling theory [19] as follows:

$$
\eta=\exp \left\{-\pi \frac{\omega}{c} L\left(\frac{\sqrt{u_{e}}}{2}\right)^{1 / 2}\left[2 \sqrt{u_{e}}\left(1-N_{\|} / N_{\|}^{o p t}\right)^{2}+N_{\perp}^{2}\right]\right\}
$$

where $L$ is the density gradient scale length and the optimum parallel refraction index for transmission is $N_{\|}^{\text {opt }}=\left(\sqrt{u_{e}} /\left(1+\sqrt{u_{e}}\right)\right)^{1 / 2}$, with all the parameters taken at the reflection point.

In the calculations with many rays, the gaussian beam is simulated with rays perpendicularly distributed over the wave front surface. The ray tracing method has a fundamental limitation when it is applied to the $\mathrm{O}-\mathrm{X}$ conversion process because it cannot take into account the beam spectrum, which is a critical issue for the $\mathrm{O}$ $\mathrm{X}$ conversion efficiency of a Gaussian beam. It has been demonstrated [20] that the optimum $\mathrm{O}-\mathrm{X}$ conversion efficiency is obtained when the beam curvature matches the curvature of the $\mathrm{O}$ mode cutoff layer. Moreover, if this condition is true, the simulation with rays perpendicularly distributed over the matched wavefront surface provides the best estimate of the $\mathrm{O}-\mathrm{X}$ conversion problem [12]. For the optimum launching direction only the central ray has maximum conversion efficiency. Any other ray has a lower conversion efficiency, which depends on its particular direction. Therefore, the total conversion efficiency of the beam is lower than $100 \%$. This is directly included in the ray tracing calculation, and thus in the final current drive calculation. As example of one ray simulation see figure 1 , where $90 \%$ O-X conversion efficiency takes place. 


\section{Current drive models}

Two mechanisms take part in the current drive by EC waves. On the one hand, the preferential heating of resonant electrons in one particular direction along the magnetic field lines, modifying asymmetrically the electron resistivity (Fisch-Boozer mechanism [21]). And, on the other hand, the diffusion of electrons from the passing to the trapping region in momentum space, leading to an asymmetry in the number of current-carrying electrons (Ohkawa mechanism [22]). The contribution to the current resulting from each of these mechanisms has opposite direction, being the latter important for large inverse aspect ratio $(\epsilon)$ in tokamaks and for large magnetic ripple in stellarators.

The calculation of the current involves the resolution of the linearised Fokker-Planck equation for the perturbed distribution function of electrons, including self-collisions, collisions with ions and the wave-induced flux $\mathbf{S}_{w}$. Techniques based on Langevin equations or Green functions have established a fast route for numerical simulations avoiding the usage of computational time-consuming Fokker-Planck codes. The high speed limit (hsl) has also been traditionally considered for the expansion of the selfcollision operator in powers of $v_{t h} / v$, where $v_{t h} \equiv \sqrt{2 T_{e} / m_{e} c^{2}}$ is the electron thermal velocity, with $T_{e}$ the electron temperature in energy units, $m_{e}$ the electron mass, and $v$ is the velocity of the current carrying electrons. Although the models based on the hsl assumption work acceptably well in most cases, its applicability is doubtful for small values of the parallel refractive index $N_{\|}$. Furthermore the hsl approximation prevents the models from fulfilling momentum conservation requirements.

\subsection{Adjoint approach formalism}

Under the adjoint approach formalism [23] three different choices for the Spitzer function $K(u)$, thus for the response (Green's) function $\chi(u, \lambda)$ (see eq. 8), can be taken into account. The parameters $u=p / m c$ and $\lambda=u_{\perp} / u^{2} b$ are the normalized momentum and the normalized magnetic moment respectively, being $b=B / B_{\max }$ the local magnetic field normalized to the maximum value in a flux surface.

First, and assuming the hsl approximation, the non relativistic expression for the Spitzer function proposed by Taguchi in Ref. [24] is,

$$
K_{\mathrm{T}}(u)=\frac{f c}{Z_{\text {eff }}+1+4 f_{c}},
$$

being $Z_{\text {eff }}$ the effective ion charge and $f_{c}$ the fraction of circulating particles in a given flux surface (see eq. 9). Secondly, the relativistic generalization of the Taguchi expression, formulated by Lin-Liu in Ref. [25],

$$
K_{\mathrm{L}}(u)=\left[\frac{\gamma(u)+1}{\gamma(u)-1}\right]^{\hat{\rho} / 2} \int_{0}^{u} d u^{\prime}\left[\frac{u^{\prime}}{\gamma\left(u^{\prime}\right)}\right]^{3}\left[\frac{\gamma\left(u^{\prime}\right)-1}{\gamma\left(u^{\prime}\right)+1}\right]^{\hat{\rho} / 2},
$$

wher $\gamma=\left(1+u^{2}\right)^{1 / 2}$ is the Lorentz factor, and $\hat{\rho} \equiv\left(Z_{\text {eff }}+1\right) / f_{c}$. 
Finally, the weakly relativistic model with momentum conservation [26, 27], denoted as $w r-m c$ model hereafter, provides the Spitzer function $K_{\mathrm{wr}-\mathrm{mc}}(u)$ as the trial function $K(u)=(u / \gamma) \sum_{i=1}^{4} d_{i} u^{i}$ that minimizes the functional

$$
\begin{aligned}
S[K]= & \int \mathrm{d}^{3} u\left[\frac{K}{f_{e}} C_{1}^{e}\left(K f_{e}\right)-\frac{f_{t r}}{f_{c}} \frac{K}{f_{e}} \nu_{e}(u) K-\right. \\
& \left.2 \nu_{0 e} \frac{u_{\|}}{\gamma} K-2 \zeta\left(u_{\|} C_{1}^{e}\left(K f_{e}\right)-\frac{f_{t r}}{f_{c}} \nu_{e}(u) K-2 \nu_{e 0} \frac{u u_{\|}}{\gamma} f_{e}\right)\right]
\end{aligned}
$$

which represents the collisional entropy production, where $C_{1}^{e}=C_{1, \mu=\infty}^{e}+\mu^{-1} C_{1,1}^{e}+$ $\mu^{-2} C_{1,2}^{e}+O\left(\mu^{-3}\right)$ is the expansion of the collision operator in a power series in $\mu^{-1}, \mu=m_{e} c^{2} / T_{e}, f_{e}=\frac{\mu}{2 K_{2}(\mu)} \mathrm{e}^{-\mu \gamma}$ is the relativistic Maxwellian, with $K_{n}(x)$ the modified Bessel function of the second kind and order $n, \nu_{e 0}=e^{4} n_{e} \ln (\Lambda) /\left(4 \pi \epsilon_{0}^{2} m_{e}^{2} v_{t h}^{3}\right)$ is the thermal electron collision frequency with $e$ and $n_{e}$ the electron charge and density, $\Lambda$ stands for the Coulomb logarithm and $\epsilon_{0}$ the vacuum permittivity, and $\nu_{e}(u) \equiv \nu_{e e}(u)+\nu_{e i}(u)$ is the total electron collision frequency given by the sum of the electron-electron plus the electron-ion collision frequencies. See Ref. [27] for the details of the calculation for the coefficients $d_{i}$ and $\zeta$ and the extension of this weakly relativistic mc model for arbitrary collisional regimes, as well as Refs. [28, 29] for the application of the model to the ECCD calculation for ITER conditions. The applicability range of this model is $0.5<u / u_{t h}<4$, although the relativistic expansion of the collision operator is bounded by a maximum momentum established by the inequality $u_{\max } / \mu \leq 1$, which in the case of the TJ-II typical electron temperature covers the whole momentum space. For the remainder of the text the non-relativistic version of this model, originally proposed by Romé [26], will be denoted as $n r-m c$ model.

For any of the expressions for the Spitzer function written above, the response function in the low collisional regime (lmfp regime), $\nu_{e} \ll \tau_{b}^{-1}$ with $\tau_{b}$ the bouncing time, is expressed in terms of $K(u)$ as,

$$
\chi(u, \lambda)=-\operatorname{sign}\left(u_{\|}\right) \frac{1}{f_{c}} H(\lambda) K(u) .
$$

where $f_{c}$ and $H(\lambda)$ are expressed as,

$$
f_{c}=\frac{3}{4}\left\langle b^{2}\right\rangle \int_{0}^{1} \frac{\lambda \mathrm{d} \lambda}{\langle\sqrt{1-\lambda b}\rangle}, H(\lambda)=\frac{1}{2} \Theta(1-\lambda) \int_{\lambda}^{1} \frac{\mathrm{d} \lambda^{\prime}}{\left\langle\sqrt{1-\lambda^{\prime} b}\right\rangle},
$$

denoting $\langle\ldots\rangle$ the flux surface averaging operation and $\Theta(x)$ the Heaviside function. $\Theta(x)=1$ for passing particles $(x \geq 0)$ and $\Theta(x)=0$ for trapped particles $(x<0)$. In the high collisional regime $\left(\nu_{e} \gg \tau_{b}^{-1}\right)$ the effective circulating particle fraction $f_{c}=1$, and the function $H(\lambda)=\left|u_{\|}\right| / u$.

Finally, the current efficiency $\eta_{\mathrm{CD}}$ in terms of the response function is given by 


$$
\eta_{\mathrm{CD}}=\frac{\left\langle j_{\|}\right\rangle}{P_{\mathrm{abs}}}=\frac{e v_{\mathrm{th}}\langle b\rangle}{\nu_{e} m_{e} c^{2}} \frac{\int \mathrm{d} u_{\|}\left[D_{\mathrm{ql}} \hat{\Lambda}\left(f_{e}\right) \hat{\Lambda}(\chi)\right]_{\gamma=\gamma_{\mathrm{res}}}}{\int \mathrm{d} u_{\|}\left[D_{\mathrm{ql}} \hat{\Lambda}\left(f_{e}\right)\right]_{\gamma=\gamma_{\mathrm{res}}}}
$$

where $\gamma_{\text {res }}=Y_{s}+u_{\|} N_{\|}$is the Lorentz factor for resonant electrons, $Y_{s}=s \omega_{c} / \omega$ with $\omega_{c}$ and $\omega$ are the electron cyclotron and wave frequencies, $D_{\mathrm{ql}}=u_{\perp}^{2}\left|\Pi_{s}\right|^{2}$ is the normalized quasi-linear Kennel-Engelmann diffusion coefficient for the harmonic $s$ [30], with the polarization factor $\Pi_{s}=e_{-} J_{s-1}\left(k_{\perp} \rho_{e}\right)+e_{+} J_{s+1}\left(k_{\perp} \rho_{e}\right)+e_{z}\left(u_{\|} / u_{\perp}\right) J_{s}\left(k_{\perp} \rho_{e}\right)$, $\hat{\Lambda}=\left(\partial / \partial \gamma+N_{\|} \partial / \partial u_{\|}\right), \rho_{e}$ the electron Larmor radius, $k_{\perp}$ the perpendicular wave vector, and $J_{n}$ the n-order Bessel function. Differently to the electromagnetic mode propagation, the EBWs are characterized by the fact that their perpendicular wave vector can present large values. Therefore, the argument of Bessel functions $k_{\perp} \rho_{e}$ can be larger than 1, thus invalidating the Larmor radius expansion and making mandatory to estimate the Bessel functions without any approximation.

\subsection{Langevin equations formalism}

The relativistic response function introduced by Fisch is based on the Langevin equations for the test particle [31]. This expression was corrected including the Ohkawa effect, thus taking into account the trapping through diffusion of initially passing particles, and it was applied to the estimation of ECCD in TJ-II [32]. The relativistic response function, without this trapping effect, is given by

$$
\chi_{\mathrm{F}}(\mathbf{u})=\frac{u_{\|}}{u}\left[\frac{\gamma(u)+1}{\gamma(u)-1}\right]^{\left(1+Z_{\mathrm{eff}}\right) / 2} \int_{0}^{u} \mathrm{~d} u^{\prime}\left[\frac{u^{\prime}}{\gamma\left(u^{\prime}\right)}\right]^{3}\left[\frac{\gamma\left(u^{\prime}\right)-1}{\gamma\left(u^{\prime}\right)+1}\right]^{\left(1+Z_{\mathrm{eff}}\right) / 2} .
$$

The Ohkawa-corrected response function is obtained by following the trajectories of the electrons in momentum space, and removing the contribution of those that starting with momentum $u$ become trapped with momentum $u_{T}$, and stop contributing to the current,

$$
\chi_{\mathrm{F}+\mathrm{O}}(\mathbf{u})=\chi_{\mathrm{F}}(\mathbf{u})-\chi_{\mathrm{F}}\left(\mathbf{u}_{T}\right)
$$

where the parallel component and module of $\mathbf{u}_{T}$ are given by,

$$
u_{T, \|}=\operatorname{sign}\left(u_{\|}\right) \mu_{t} u_{T} \quad ; \quad u_{T}=\frac{2 \sqrt{g(u)}}{1-g(u)} \text { and } g(u)=\frac{\gamma+1}{\gamma-1}\left(\frac{\mu_{t} u}{\left|u_{\|}\right|}\right)^{2 /\left(Z_{\mathrm{eff}}+1\right)}
$$

being $\mu_{t}=(1-b)^{1 / 2}$ the trapping parameter. This value of the momentum $u_{T}$ at which the particle becomes trapped is obtained by estimating the intersection of the loss cone and the particle trajectory in momentum space under the effect of cyclotron resonance heating and collisions. Note that equation (11) corresponds to the response function using the Lin-Liu model after ignoring the frictional trapping effects, i.e. $f_{c}=1$, 
and taking $H(\lambda)=\left|u_{\|}\right| / u$. Therefore, this model provides an upper bound to the parallel current value applicable in the collisional limit [33].

In order to obtain this upper bound to the current calculation as well as the estimation of the possible Ohkawa contribution to the current, this model is also present in the calculation in next sections. Finally the current efficiency $\eta_{\mathrm{CD}}$ differs from expression (10) in the preceding constant, where in the present case is $A=e / \nu_{e 0} m c$, due to a different definition of the dimensionless response function in the adjoint approach and Langevin equations formalisms. The contribution of trapped particles $\left(u_{\|} / u \leq \mu_{t}\right)$ is also removed from the current integral. 


\section{Numerical results}

The calculation of the parallel current drive efficiency $\eta_{\mathrm{CD}}$ has been carried out adapting the different models reviewed in section 3 to the ray tracing code TRUBA. The parallel current generated at a given point is assumed to be distributed uniformly on the magnetic surface where it was generated, or equivalently the parallel transport is assumed to be infinite. The current carriers are assumed to not leave the flux surface where they were created and to distribute quickly on that surface. Although this point of view does not take into account the radial transport, whose analysis is out of the scope of this paper, note that it does not affect the comparison between the models since all results are obtained under the same assumption. Furthermore, the contributions to the same flux surface must be summed whenever a given ray comes across it more than once.

The total toroidal current element between two flux surfaces separated a $\Delta \rho$ and enclosing a surface $\Delta S(\rho)$ is expressed in terms of the parallel current density $J_{\|}(\rho)$ as : $\Delta I_{\mathrm{CD}}(\rho)=J_{\|}(\rho)\left\langle B_{\phi} / B\right\rangle(\rho) \Delta S(\rho)$. The parallel current density $J_{\|}$is weighted by $\left\langle B_{\phi} / B\right\rangle$ for experimental reasons: since the measurement of the toroidal current, in particular in the TJ-II, involves the presence of a Rogowski coil surrounding the plasma at a given toroidal plane, the relevant quantity to be measured is the projection of the parallel current density on the toroidal surface. This requirement is necessary for a 3D magnetic structure, since the parallel direction varies strongly from one point to another of the same flux surface. The usage for this purpose of the value of $B_{\phi} / B$ at the point where the wave interacts with the electrons would break the dependence of $\Delta I_{\mathrm{CD}}$ on only the flux surface, thus the integration over $\rho$ be doubtfully evaluated. Hence, the presence of the flux surface averaging acting on $B_{\phi} / B$. The value of $\left\langle B_{\phi} / B\right\rangle(\rho)$ in TJ-II monotonically grows from 0.85 , at $\rho=0$, to 0.95 , at $\rho=1$, thus it corrects in approximately a $10 \%$ the current value obtained without taking it into account.

The 3D geometry of the TJ-II is also taken into account by considering the 3D VMEC coordinates Jacobian, required for flux surface averaging, and it is provided by a numerical library [34]. This library makes use of 50 flux surfaces between $\rho=0$ and

$\rho=1$. The volume enclosed by a given flux surface is fitted by $V(\rho) \approx 1.107 * \rho^{2}$ for the standard TJ-II magnetic configuration, which is the one considered in this work. Thus, a numerical inaccuracy for the volume arises, not from the volume estimation itself, but for the estimation of $\rho$ at any point of the ray expressed in Cartesian coordinates. This error provokes, in the estimation of the volume enclosed between two consecutive ray steps $(\Delta V)$, a noise that is of the same order of magnitude as $\Delta V$ when the ray is almost tangent or is closed to the magnetic axis. In order to remove the noise, $\Delta V$ is smoothed, and an lower bound for $\Delta V$ is established, $\Delta V_{\min }=2 \times 10^{-4} \mathrm{~m}^{-3}$, that corresponds to the volume enclosed by the flux surface $\rho \approx 0.13$.

As it is said above, the calculation has been performed considering the standard TJ-II magnetic configuration, and the optimum beam injection in terms of maximum O-X transmission efficiency [12]. A total injected power of $300 \mathrm{~kW}$ before O-X 
conversion is simulated. The shape of the density and temperature profiles are given by $n_{e}(\psi)=n_{0}\left(1-\psi^{2}\right)^{7}$ and $T_{e}(\psi)=T_{0}\left(1-\psi^{7}\right)^{10}$ respectively, with $n_{0}$ and $T_{0}$ the central values and $\psi$ the normalized magnetic flux. These profiles are similar to the measured experimentally by Thomson Scattering in the NBI high density regime of TJ-II. Note, that since one ray simulations do not take into account the refractive index spectral density and the width of the beam, it is assumed that it will not provide neither the most realistic absorbed power density nor current density profiles. In any case, they can be used for studying the current drive models validity ranges and check the resonance condition domain in momentum space along one ray. This is what is done next in subsection 4.1 before the results for multi-ray simulations are presented.

\subsection{Current drive efficiency and density for one ray calculation}

Figure 2 shows the absolute value of the current drive efficiency $\left|\eta_{\mathrm{CD}}(l)\right|$ for each model and the absorbed power density $P_{\text {abs }}(l)$ as a function of the length $l$ travelled by the mode converted ray. The total toroidal current $I_{\mathrm{CD}}$ resulting from each model are shown in the legend. The figure corresponds to the single-ray simulation represented in figure 1 , thus $T_{0}=0.4 \mathrm{keV}$ and $n_{0}=5 \times 10^{19} \mathrm{~m}^{-3}$. The corresponding parallel current density along that ray is plotted in figure 3. First, the Fisch and Ohkawa-corrected Fisch models curves are almost overlapped, which indicates that the Ohkawa contribution is negligible. This is due to the high value of $\left|N_{\|}\right|$where power is deposited, in the range of 0.5. Fisch models also estimate a current density higher than the Taguchi and Lin-Liu models, since the latter ones take into account the frictional reduction of the circulating electrons with the trapped population. The difference between the Taguchi and Lin-Liu models comes from the relativistic effects, that the latter includes, and that can be observed also comparing the weakly and non relativistic mc models. Finally, independently on the collisional regime considered by the hsl models, these provide a lower value than the mc models.

Figure 4 represents the normalized radius $\rho, Y_{s=1}$ and $N_{\|}$along the ray path, as well as the corresponding resonance condition domain in $u_{\|}$(coloured area delimited by the black thick line). The mc model validity range is also represented by the horizontal non-delimited stripes. Strictly speaking, a non-zero current drive efficiency value is obtained, as long as the resonance condition exists, and this value increases with the momentum of the resonant electrons. This can be observed comparing $\left|\eta_{\mathrm{CD}}\right|$ in figure 2 and the resonance condition domain in figure 4 just before/after the resonant condition offset/onset, or between $l=0.25 \mathrm{~m}$ and $l=0.3 \mathrm{~m}$. On the other hand, the absorbed power density in such intervals in momentum space is negligible, if not identically zero, since there are almost no electrons, and the current drive efficiency, although existing, does not result any current. Thus, focusing on the region where the beam power is deposited, from $l=0.3 \mathrm{~m}$ on (see figure 2) and concerning the validity of each model in the same interval (see figure 4), it is shown that the efficiency of the mc models is estimated slightly out of its validity range just before the resonance appears. Despite 
of this, the result provided by the model is still valid, since in that region the power deposited is low. Once the calculation is carried out within its range of validity, and comparing it with the Taguchi and Lin-Liu results, the mc (weakly or non-relativistic) and hsl models (Taguchi, Lin-Liu and Fisch) agree in the same descending trend for the efficiency along the ray length. In the end of the power density deposition profile, and concerning the usage of the hsl-based models, it can be observed how low energetic electrons $\left(u_{\|} \lesssim 2 u_{t h}\right)$ are involved in the current generation, and thus the hsl-assumption may not be fulfilled. Finally, comparing the Fisch and Lin-Liu models, the total toroidal current value (see legend of figure 2) shows approximately a 10 percent difference, while comparing Lin-Liu and weakly relativistic mc models this difference is around a 20 percent. Thus, the choice of the model type, hsl or mc, seems to be more important than the collisional regime in TJ-II. In this particular case, when both hsl and mc models come up against its limits of application, the amount of power density is low, and thus no significant deviation from a coherent value is expected. But it should be noted that, although hsl and mc models may step inside a region where its applicability is dubious, $u_{t h} / u \lesssim 1$ and $0.5<u / u_{t h}<4$ respectively, the mc models are better positioned, since the result is weighted by the electron population, which over $4 u_{t h}$ is almost negligible.

Although the example illustrated in figures 2 and 4 does not allow drawing any general conclusion, the previous analysis provides an idea of the phenomenology that each model can find in momentum space, and how their applicability is threaten in some regions, although absorbed power density is low. Nevertheless, such detailed analysis in the case of multi-ray simulation, which is mandatory for a correct current estimation, would result a tedious task outside the scope of this work. Figure 5 highlights the necessity of multi-ray simulations in order to reach the convergence of the current density profile $J_{\|}(\rho)$ and, hence, to get a correct total toroidal current value. The figure shows the parallel current density profile $J_{\|}(\rho)$ calculated using the weakly relativistic mc model for a different number of rays used for the beam simulation. In the top right quadrant of the figure the convergence of the current density profile is shown quantitatively. The array of number of rays considered is $\mathbf{N}=(1,5,17,41,81,121)$, so that the distance between to profiles with consecutive number of rays $N_{i}$ and $N_{i-1}$ can be defined as: $d\left(J_{\| N_{i}}, J_{\| N_{i-1}}\right)=\int_{0}^{1} \mathrm{~d} \rho\left|J_{\| N_{i}}(\rho)-J_{\| N_{i-1}}(\rho)\right|$. This distance is normalized to the distance between the profiles provided by the runs launching 1 and 5 rays, i.e. $d\left(J_{\| N_{2}}, J_{\| N_{1}}\right)$, to define what it is actually plotted: $D_{i}=d\left(J_{\| N_{i}}, J_{\| N_{i-1}}\right) / d\left(J_{\| N_{2}}, J_{\| N_{1}}\right)$. As it can be observed, 121 rays simulation reduces in a factor of 10 this normalized distance $D_{i}$ between profiles, which together with the good convergence of the total current value (see legend) makes this choice suitable for a convincing simulation. For simplicity, the trajectories of 20 of these 121 rays are plotted in figure 6 . The black thick lined ray represents the central ray. 


\subsection{Multi-ray simulation scanning $n_{0}$ and $T_{0}$}

In the present section, a comparison of the total toroidal current $I_{\mathrm{CD}}$ varying the central value of the profiles written above for the electron temperature and density is discussed, as well as the importance of considering a relativistic model, and the contribution of the Ohkawa mechanism to the driven current. For each run, the beam is simulated with 121 rays. For each map eight values of $T_{0}$ between 0.2 to $1.6 \mathrm{keV}$ and twenty one in $n_{0}$ between 2.0 and 7.0 in units of $\times 10^{19} \mathrm{~m}^{-3}$ have been taken into account.

The first of these maps, figure 7 , shows the total toroidal current $I_{\mathrm{CD}}$ using the LinLiu model, while figure 8 shows the same for the relativistic me model. Contour lines every $2 \mathrm{kA}$ are also represented. Both models manifest a clear increasing behaviour of the driven current with the temperature up to $T_{0}=1 \mathrm{keV}$. After that central temperature the current decreases up to negative values. This is due to the change in the radial profile of power deposited and current generated. Up to $T_{0}=1 \mathrm{kev}$, the current density is localized in central and mid values of $\rho$, where positive contribution to the total current are found. From $T_{0}=1 \mathrm{kev}$ on, the central contribution dissapears, and the total current is a result of the positive mid-radial contribution and an external negative one, that becomes the main contribution to the current as $T_{0}$ increases. This behaviour is shown, using the weakly relativistic me model, in figure 9 , where the parallel current density profiles are represented for different central temperatures and a constant central density of $n_{0}=4.0 \times 10^{-19} \mathrm{~m}^{-3}$. The reason why a negative current contribution does not appear in the edge for low temperatures is due to the fact that in the first part of the transmitted ray, the resonance condition extends to momentum values where there are almost no electrons, thus no damping, nor power absorption and current. As temperature increases the resonance condition moves towards the centre of momentum space, where a larger electron population leads to an appreciable power deposition and subsequently negative current generation, since $N_{\|}>0$ and $\eta_{\mathrm{CD}}<0$. The evolution of the resonance condition with the temperature, as well as the power deposition at the entrance of the transmitted ray, can be observed in the set of figures 10. For this figure, the temperatures considered are the same than those for the current density profiles represented in figure 9 , i.e. $T_{0}=\{0.4,0.8,1.2,1.6\} \mathrm{keV}$. The resonance condition domain is represented by the coloured area delimited by a solid line. The effective radius $\rho$ and $N_{\|}$are also plotted. It can be noticed for the case $T_{0}=1.6 \mathrm{keV}$ that, despite the power deposition at the first part of the transmitted ray path is lower than in the more internal second deposition area, at the former, the negative current drive efficiency $\eta_{\mathrm{CD}}$ is larger in modulus than in the latter, and that is why the total current results lower than zero. This can be observed in figure 11, where the current drive efficiency along the central ray of the multi-ray simulation used for the case $T_{0}=1.6 \mathrm{keV}$ in figure 9 is represented using the weakly relativistic mc and Lin-Liu models. Returning to figures 7 and 8, the Lin-Liu model shows a less peaked and slightly lower profile than the mc model. In the TJ-II, where the electron temperature is not expected to exceed $1 \mathrm{keV}$ in the NBI discharges where EBW heating system will operate, both models indicate the 
possibility of a toroidal current generation between 1 to $10 \mathrm{kA}$.

Regarding whether or not a relativistic model should be used, figure 12 shows the total current drive efficiency as a function of $T_{0}$ for different central densities, and comparing the weakly relativistic and non-relativistic mc models. The total current drive efficiency is defined as $I_{\mathrm{CD}}\left\langle n_{e}\right\rangle R_{0} / P_{\mathrm{EC}}$, where $\left\langle n_{e}\right\rangle$ is the line electron density, $R_{0}=1.5 \mathrm{~m}$ is the TJ-II major radius, and $P_{\mathrm{EC}}$ is the injected power, $300 \mathrm{~kW}$ as said above. It is intuitively clear the relativistic effects become more important as the temperature increases, and this is what figure 12 shows below $T_{0}=1.0 \mathrm{keV}$. Again, since $I_{\mathrm{CD}}$ is a composition of contributions with different sign at higher temperatures, as figure 9 shows, the increasing difference with the temperature between the current density profiles provided by each model, is concealed in the value of $I_{\mathrm{CD}}$. Despite of this, an appreciable difference in the result exists. Respecting the total current efficiency value itself, and focusing in the TJ-II, even the lowest value of total EBCD efficiency shown in the figure 12 is higher than the one measured in ECCD experiments: $I_{\mathrm{CD}}\left\langle n_{e}\right\rangle R_{0} / P_{\mathrm{EC}} \approx 0.001$ in unit of $10^{20} \mathrm{Am}^{-2} \mathrm{~W}^{-1}$ (see ref. [35]), and shows how efficient EBWs can be for generating current.

Finally, the same scan in $T_{0}$ and $n_{0}$ of the toroidal current $I_{\mathrm{CD}}$ is represented for the Ohkawa-corrected Fisch model in figure 13. As it is observed, it is the less peaked of these maps, and $I_{\mathrm{CD}}$ deviates from the value provided by the Lin-Liu and weakly relativistic mc models noticeably. The highest contour line in the figure corresponds to the value of $16 \mathrm{kA}$, while in the figure corresponding to the Lin-Liu model (fig. 7) it does to the value of $14 \mathrm{kA}$. The separation between contour lines is $2 \mathrm{kA}$. Therefore, comparing figures 7 and 13 a difference of around $2 \mathrm{kA}$ exists in the half bottom part of the maps, while in the upper part of figure 13, the high density of contour lines observed shows that at high temperature the disagreement can reach about $10 \mathrm{kA}$. This disagreement is due to ignoring the frictional reduction of the current. In the high collisional regime, assumed in the Fisch model, the circulating particle fraction is taken as $f_{c}=1$. And, at high temperature, where the current generation is localized at external radial positions (see figure 9), this particle fraction deviates strongly from the value of one. Concerning the Ohkawa contribution, this is negligible, as it can be seen in figure 14, where the total toroidal current efficiency is represented as a function of the temperature, comparing the Fisch model with and without the Ohkawa effect. Previously, in the legend of figure 2 this small difference was expressed for a single ray simulation in the total current drive value. This negligible influence is due to the high value $\left|N_{\|}\right|$at which Bernstein waves interact with the electrons (see figure 4), that displaces the resonance condition away from the trapping cone and provides the electrons with a high parallel momentum that prevent them becoming trapped.

\subsection{Multi-ray simulation varying the launching direction}

Finally, in order to count on a complete characterization of the current generated at the different launching directions that the TJ-II EBW heating system covers, in the present 
section, a scan in the positioning angles of the focusing mirror of the system is studied. The angles $\alpha_{\theta}$ and $\alpha_{\phi}$ that fix the mirror position define the poloidal and toroidal launching direction respectively. The optimum mirror position, in terms of maximum O-X transmission efficiency, see ref. [12], is expected to lay between $-34^{\circ}$ and $-32^{\circ}$ for $\alpha_{\theta}$, and for $\alpha_{\phi}$ between $-31.5^{\circ}$ and $-29^{\circ}$. In the previous section, the position of the mirror angles values taken were: $\alpha_{\phi}=-31.1^{\circ}$ and $\alpha_{\theta}=-33^{\circ}$. Around this position is theoretically expected to get a maximum $\mathrm{O}-\mathrm{X}$ conversion efficiency, although the exact one depends on the height and shape of the density and temperature profiles. The calculation of the toroidal current has been performed, tracing 121 rays per beam, and scanning each angle along 20 values in the vicinity of the optimum position. The profile is shaped as in previous sections, and the central values are the same as those taken for figures from 2 to 6 : $T_{0}=0.4 \mathrm{keV}$ and $n_{0}=5.0 \times 10^{19} \mathrm{~m}^{-3}$.

Figures 15 and 16 show the total toroidal current generated using the weakly relativistic mc and Lin-Liu models respectively. The value provided by the former one is again greater than in the Lin-Liu model, and in both cases, as expected, the O-X conversion efficiency determines the value with maximum toroidal current, localized at the theoretically optimum launching angles. The weakly relativistic mc model is again more peaked than the Lin-Liu model, whose contour lines are spread out to the left side of the figure. The current maximum values are respectively 4.5 and $3.5 \mathrm{kA}$. Finally, figure 17 represents the same map for the Fisch Ohkawa-corrected model that, compared to the Lin-Liu model, shows a difference of around $2 \mathrm{kA}$, again resulting from the lack of frictional trapping effects in the former model.

Once the heating system is under operation, a comparison of the experimental current with these results will be mandatory to know how far we are from the low (e.g. Lin-Liu or wr-mc models) or high collisional regimes (Fisch models). Since these limits provide lower and upper bounds to the current value, in principle it cannot be assured how close the experimental result will from one value or the other. It looks like that at high temperature the models applicable in the $l m f p$ regime are supposed to provide more accurate current values than the Fisch model. As a matter of fact the Fisch model gives an abnormally high current value provided at high temperatures (see figure 13). But as long as the collisional regime is not quantitatively establish no choice can be done in this direction. 


\section{Summary and future work}

The implementation of a tool for the EBCD efficiency calculation by means of different linear models implemented to the ray tracing code TRUBA has been successfully carried out. The analysis in momentum space along one ray simulation shows how the changing EBW parallel refractive index may impact on the correctness of the application of these models, as it occurs with the mc model when $N_{\|}$is high enough to include in the current integral electrons with $u>4 u_{t h}$, or in the hsl models when the $N_{\|}$is low and the wave heats almost bulk electrons. In principle, the mc models are better positioned than the hsl models, not only for self-consistency, but also for the weight of the distribution function in the momentum space region where its application is restricted. Opposite to the case of the models based on the hsl assumption.

Concerning the electron temperature and density that the EBW heating system is expected to encounter, a scan in their central values has been made, considering typical profiles from NBI-heated TJ-II plasmas. It is important to notice that the current value does not depend only on the height of the profiles but also on the change of the radial power deposition [12] and the sign of $N_{\|}$where absorption takes place. The total EBCD shows a decreasing behaviour with the density. Concerning the temperature, the trend is rather more complicated, since the current density profile changes strongly with this parameter, becoming the total EBCD a result from summing up contributions with opposite sign, and making it becomes even negative. Thus an increasing value of the total current with $T_{0}$ is obtained below $1 \mathrm{keV}$, and after that value the current decreases up to negative values as the negative contribution becomes the most important.

The conclusions that can be drawn from the models comparison are summarized as follows: first, by comparing the Fisch model with and without the Ohkawa effect included, this contribution to the total EBCD is negligible in the TJ-II. Although this fact depends on the device, it is also a feature of the high value of $\left|N_{\|}\right|$archived by the Bernstein waves. Secondly, the comparison between the results provided by the weakly relativistic and non relativistic mc models has shown that the relativistic effects, although considerable, are not decisive in the toroidal current value. The main difference among the toroidal current values provided by each model appears when the frictional reduction of the current is included or not (Lin-Liu and Fisch models respectively), as well as by demanding or not momentum conservation (see comparison between weakly relativistic and Lin-Liu models). In both cases the difference found between the current drive values can be of the order of magnitude of the current value itself.

Finally, the toroidal current is not strongly affected by the launching direction, apart from the change of the transmission efficiency with the distance to the mirror position that optimizes it. This kind of maps can provide a tool for the optimization of the electron heating without inducing a current that may damage the magnetic configuration, unless another procedure for inducing current in the plasma is provided.

Future work will cover the comparison of the results here presented with the experimental data obtained when the heating system is operating, and will enable an 

experimental test of the models here studied and the hypothesis assumed. 


\section{References}

[1] Fisch N J Rev. Modern Phys. 198759175

[2] Prater R Phys. Plasmas 2004112349

[3] Erckmann V and Gasparino U Plasma Phys. Control. Fusion 1994361869

[4] Hender T C et al Nucl. Fusion 200747 S128

[5] Prater R et al Nucl. Fusion 2008481

[6] I B Bernstein Phys. Rev. 195810910

[7] Litvak A G, Suvorov E V and Tokman M D Phys. Lett. A 199418864

[8] Laqua H P Plasma Phys. Control. Fusion 200741 R1-R42.

[9] Laqua H P et al Phys. Rev. Lett. 200390075003

[10] Alejaldre C et al Fus. Tech. 199017131

[11] Fernández et al Fusion Eng. and Design 200574325

[12] Castejón et al Nuclear Fusion 200848075011

[13] Preinhaelter J and Kopecky V Journal of Plasma Phys. 1973101

[14] Tereshchenko M A et al 2003 Proceedings of the 30th EPS Conference on Contr. Fusion and Plasma Phys. 27A P-1.18

[15] Tokman M D, Westerhof E and Gavrilova M A 2000 Plasma Phys. Control. Fusion 4291

[16] Brambilla M 1998 Kinetic Theory of Plasma Waves, Homogeneous Plasmas (Oxford: Oxford University Press)

[17] Shkarofsky I P 1966 Phys. Fluids 9561

[18] Timofeev A V 2001 Plasma Physics Reports 27922

[19] Mjølhus E J 1984 J. Plasma Phys. 317

[20] Köhn A et al 2008 Plasma Phys. and Control. Fusion 501

[21] Fisch N J and Boozer A H Phys. Rev. Lett. 198045720

[22] Ohkawa T General Atomics Report GA-A13847 1976

[23] Antonsen Jr. T M and Chu K R et al Phys. Fluids 1982251295

[24] Taguchi M Plasma Phys. Control. Fusion 198931241

[25] Lin-Liu Y R, Chan V S and Prater T Phys. Plasmas 2003104064

[26] Romé M et al Plasma Phys. and Control. Fusion 199840511

[27] Marushchenko N B, Beidler C D and Massberg H Fusion Sci. Technol. 200950180

[28] Marushchenko N B, Massberg H and Turkin Y Nucl. Fusion 200848054002

[29] Marushchenko N B, Massberg H and Turkin Y Nucl. Fusion 200949123801

[30] Kennel C F and Engelmann F 1966 Phys. Fluids 92377.

[31] Fisch N J Phys. Rev. A 1981243245

[32] Castejón, Alejaldre C and Coarasa J A Phys. Fluids B 199243689

[33] Lin-Liu Y T and Miller R L Phys. Plasmas 199421666

[34] Tribaldos V, van Milligen B P and López-Fraguas A L Informes Técnicos del Ciemat 9632001

[35] Fernández A et al 2007 Fusion Sci. Technol. 531 

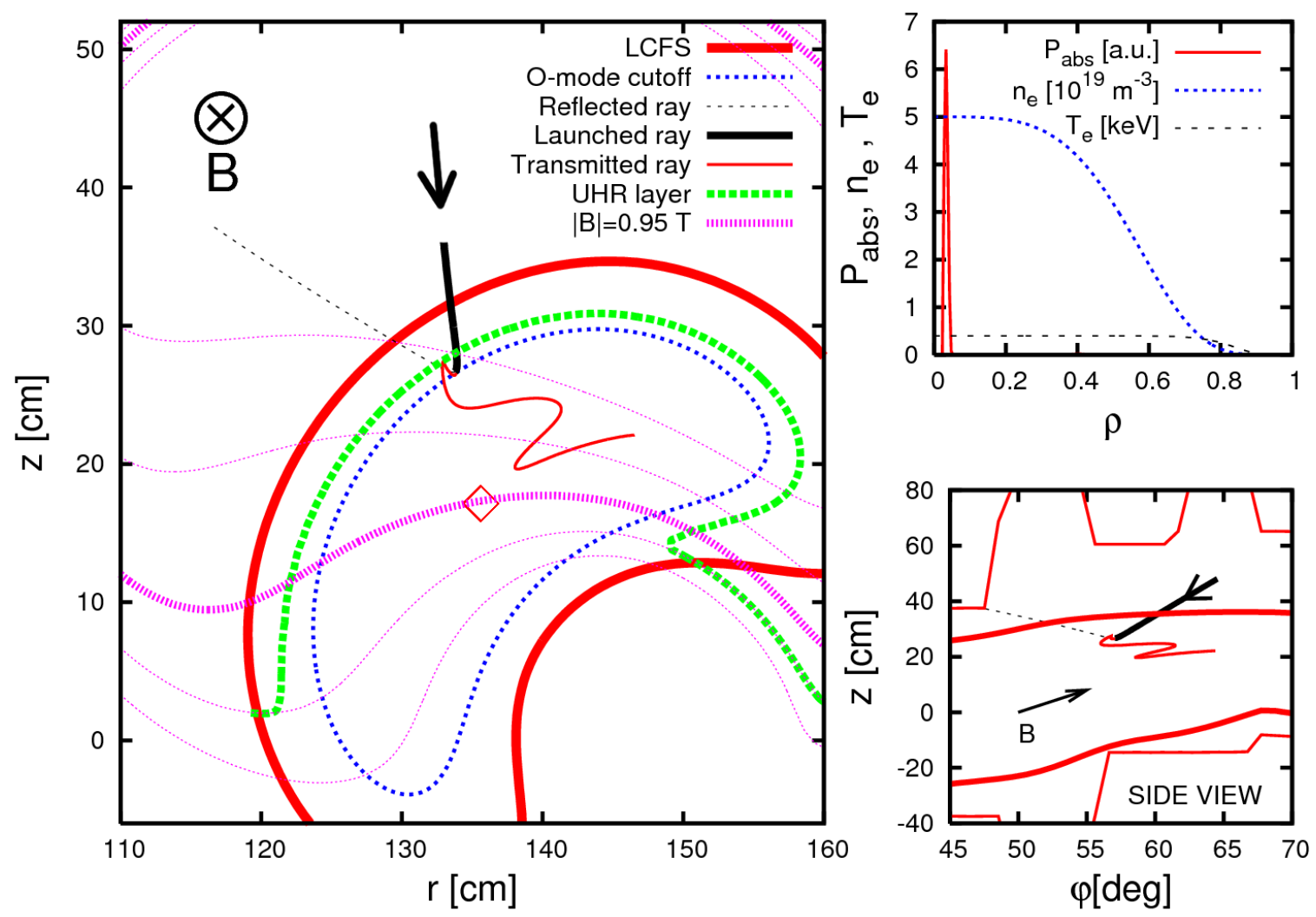

Figure 1. One ray simulation with transmission efficiency of 0.90 . Density and temperature radial profiles given by $n_{e}(\psi)=5.0\left(1-\psi^{2}\right)^{7}$ in units of $10^{19} \mathrm{~m}^{-3}$ and $T_{e}(\psi)=0.4\left(1-\psi^{7}\right)^{10} \mathrm{keV}$. On the left, the launched, reflected and transmitted rays trajectories projected in the toroidal TJ-II plane $\phi=57^{\circ}$. Contour lines of the magnetic field strength with a separation of $0.05 \mathrm{~T}$ are also represented. On the bottom right, a side view of these rays, and in the top right, the absorbed power, temperature and density profiles as a function of the normalized radius $\rho$. 


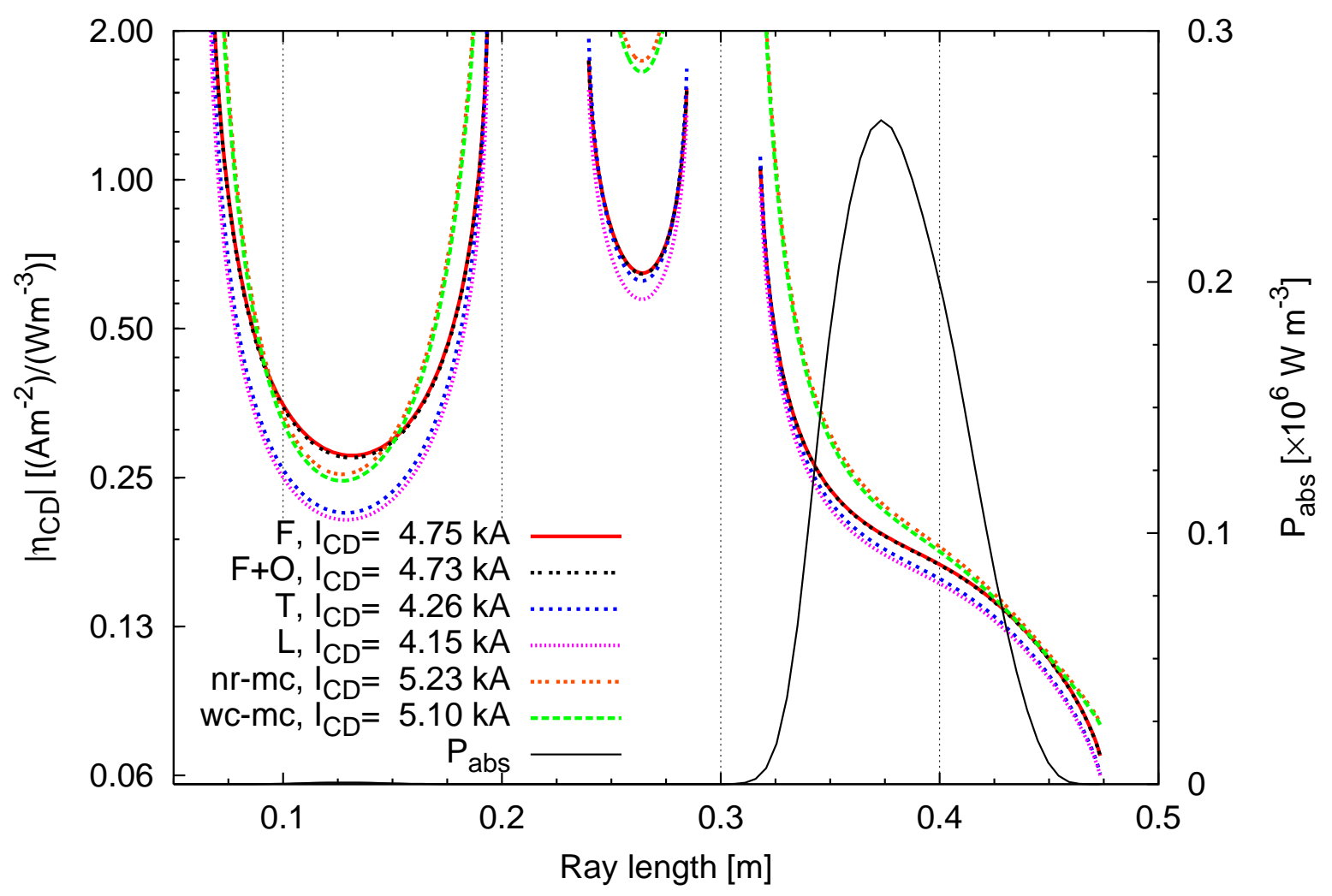

Figure 2. Current drive efficiency $\eta_{\mathrm{CD}}$ for the models considered in this work, and absorbed power density, as functions of the length traveled by the mode-converted Berstein ray. $F$ denotes the Fisch model, $F+O$ the Ohkawa-corrected version of the model, $T$ denotes the Taguchi model, $L$ the Lin-Liu one, while $w r-m c$ and $n r-m c$ are respectively the weakly and non-relativistic models based on momentum conservation. The central density and temperatures in this case are $5.0 \times 10^{19} \mathrm{~m}^{-3}$ and $0.4 \mathrm{keV}$. 


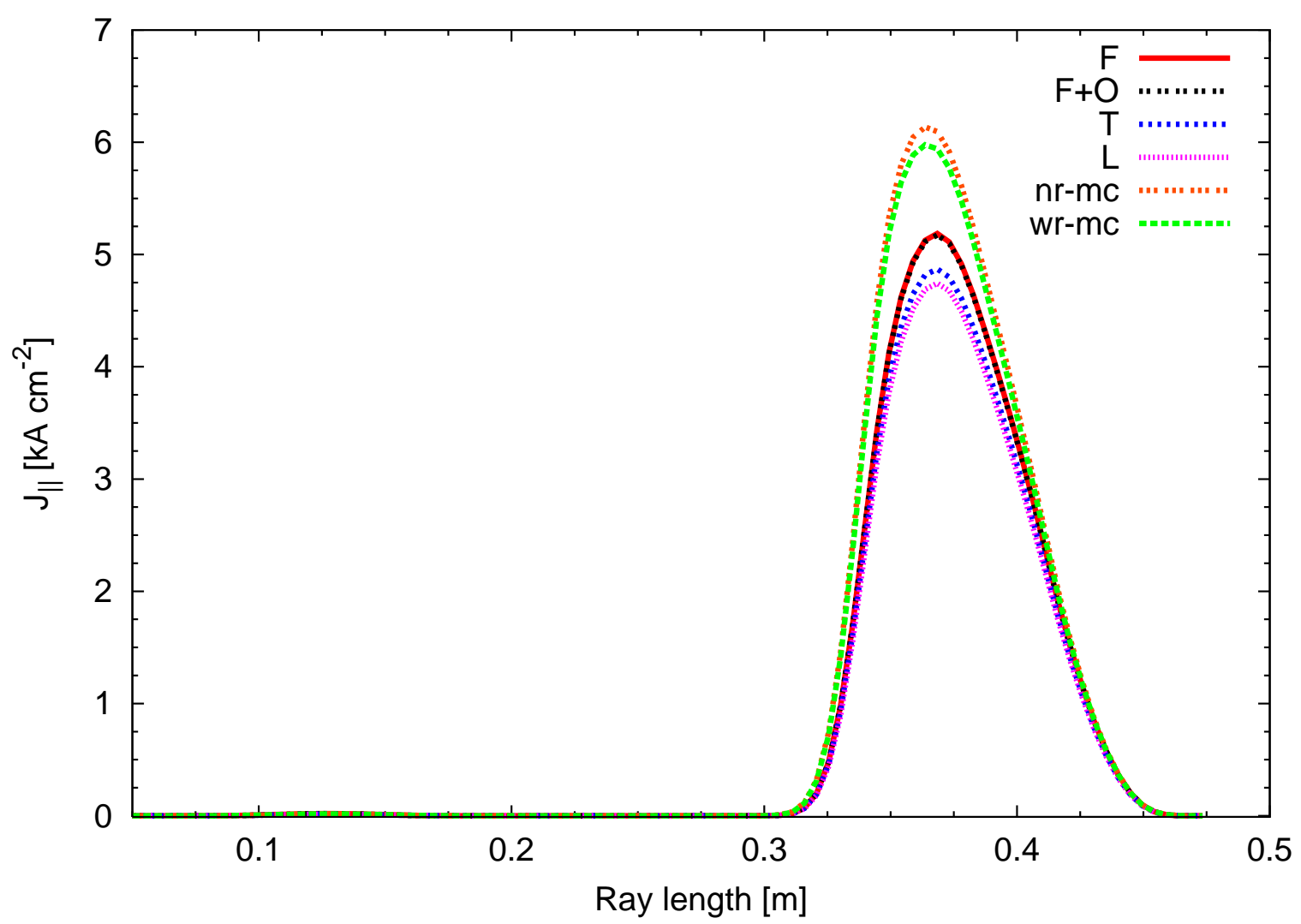

Figure 3. Parallel current density $J_{\|}$as function of the distance travelled by the transmitted ray corresponding to the models considered in figure 2 . 


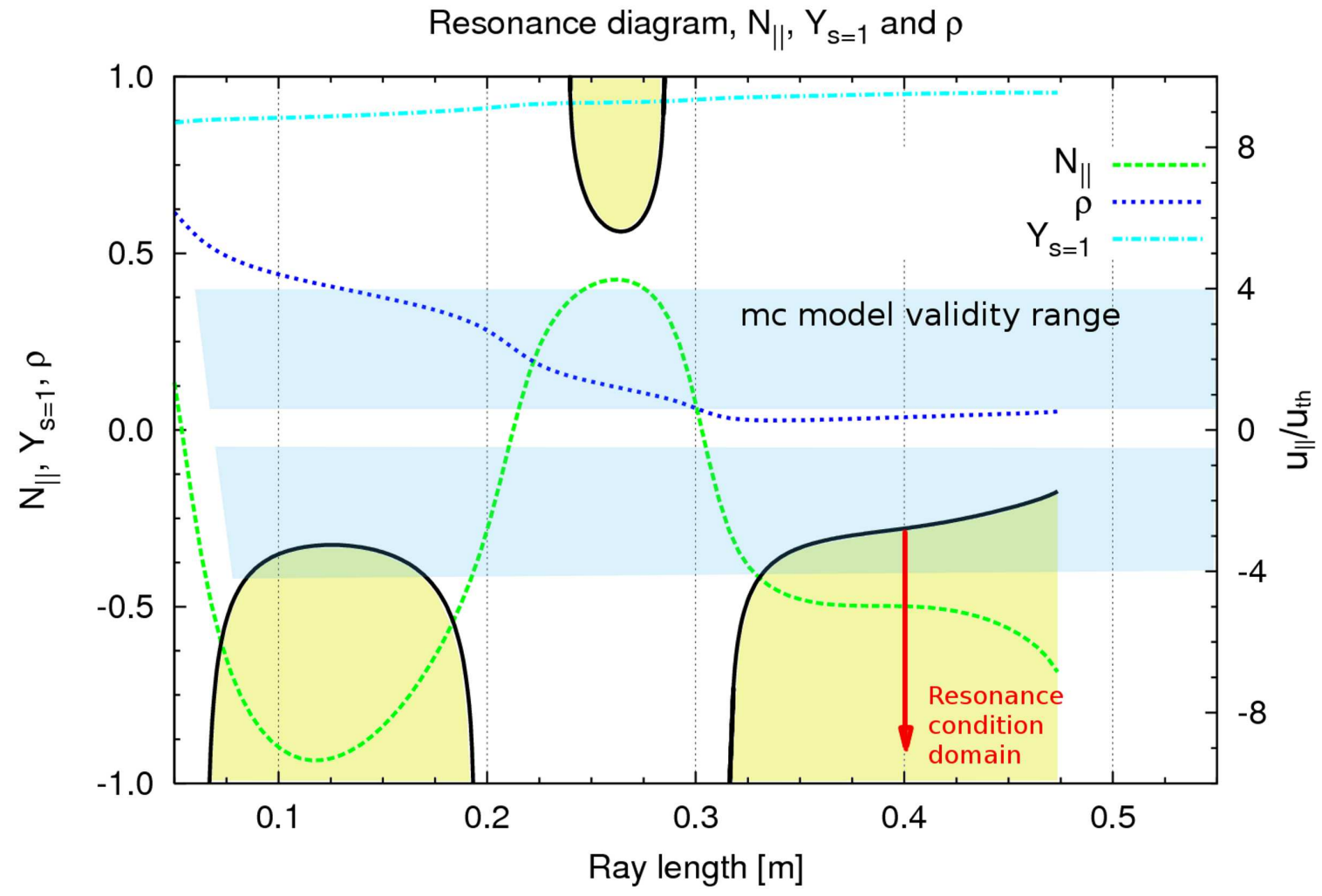

Figure 4. In the left y-axis, $Y_{s=1}$, normalized radius $\rho$ and parallel refractive index $N_{\|}$corresponding to the case represented in figure 2. Referred to the right y-axis, the area delimited by the continuous line represents the $u_{\|}$resonance condition domain along the ray path, normalized to the thermal momentum $u_{t h}$. The two non-delimited stripes represent the mc models validity range. 


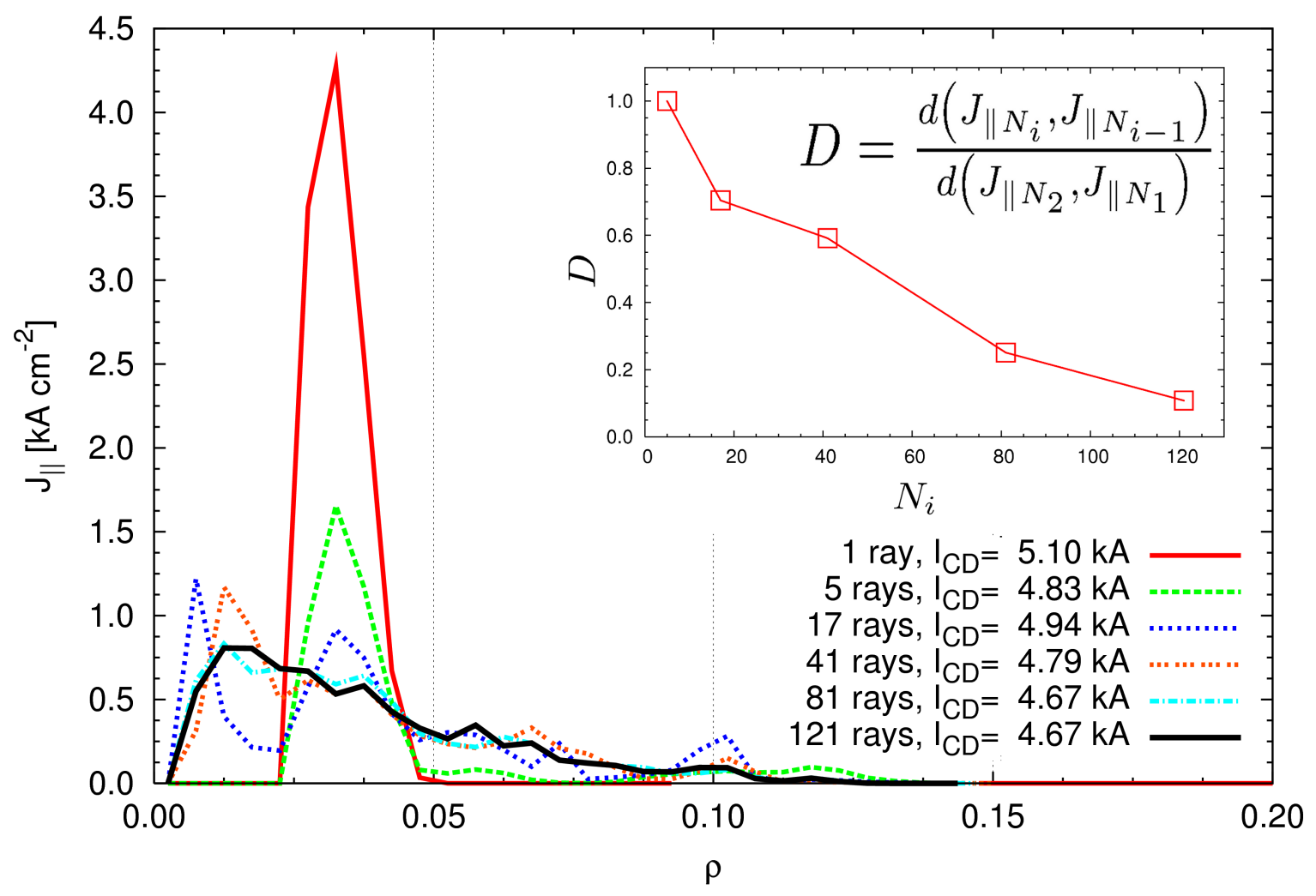

Figure 5. Radial profile of the parallel current density $J_{\|}(\rho)$ provided by the relativistic mc model, for different number of rays traced, and for the same plasma temperatures and density profiles than those used in figures 2 and 4 . The subfigure in the top right quadrant represents the distance between the profiles provided by consecutive runs sorted in ascending order by the number of rays used, $d\left(J_{\| N_{i}}, J_{\| N_{i-1}}\right)$, normalized to the distance between the first two, $d\left(J_{\| N_{2}}, J_{\| N_{1}}\right)=d\left(J_{\| 5}, J_{\| 1}\right)$. 

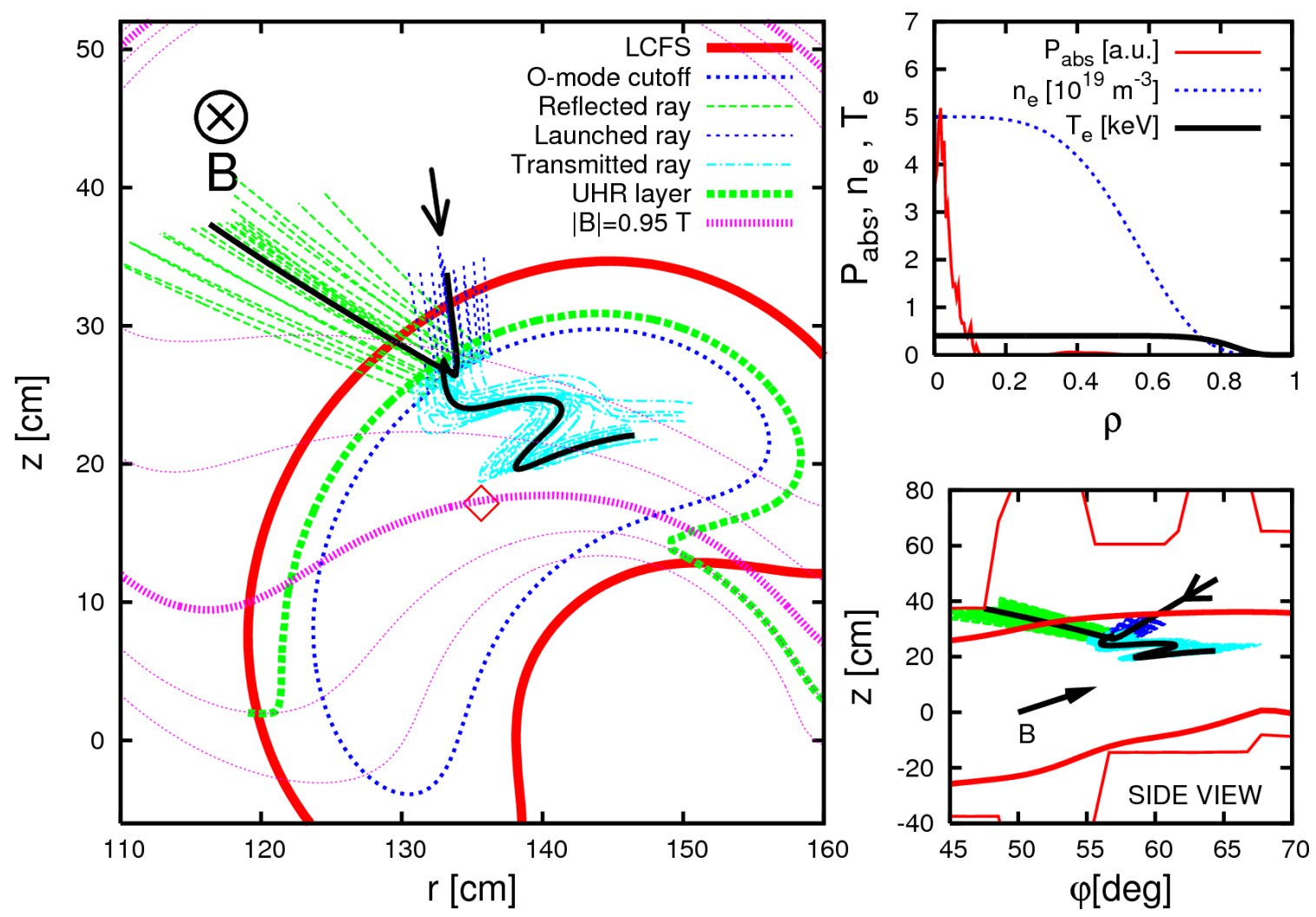

Figure 6. Trajectories of 20 of the 121 rays used for the beam simulation that provides the corresponding current density profile shown in figure 5 (Left). The arrow represents the launching direction. The Upper Hybrid Resonance layer, the O mode cut-off layer the last closed magnetic surface (LCFS) and constant B field layers are also represented. The power deposition, density an temperature profiles are shown in the top right figure and a side view of the same rays is plotted in the right bottom panel. 


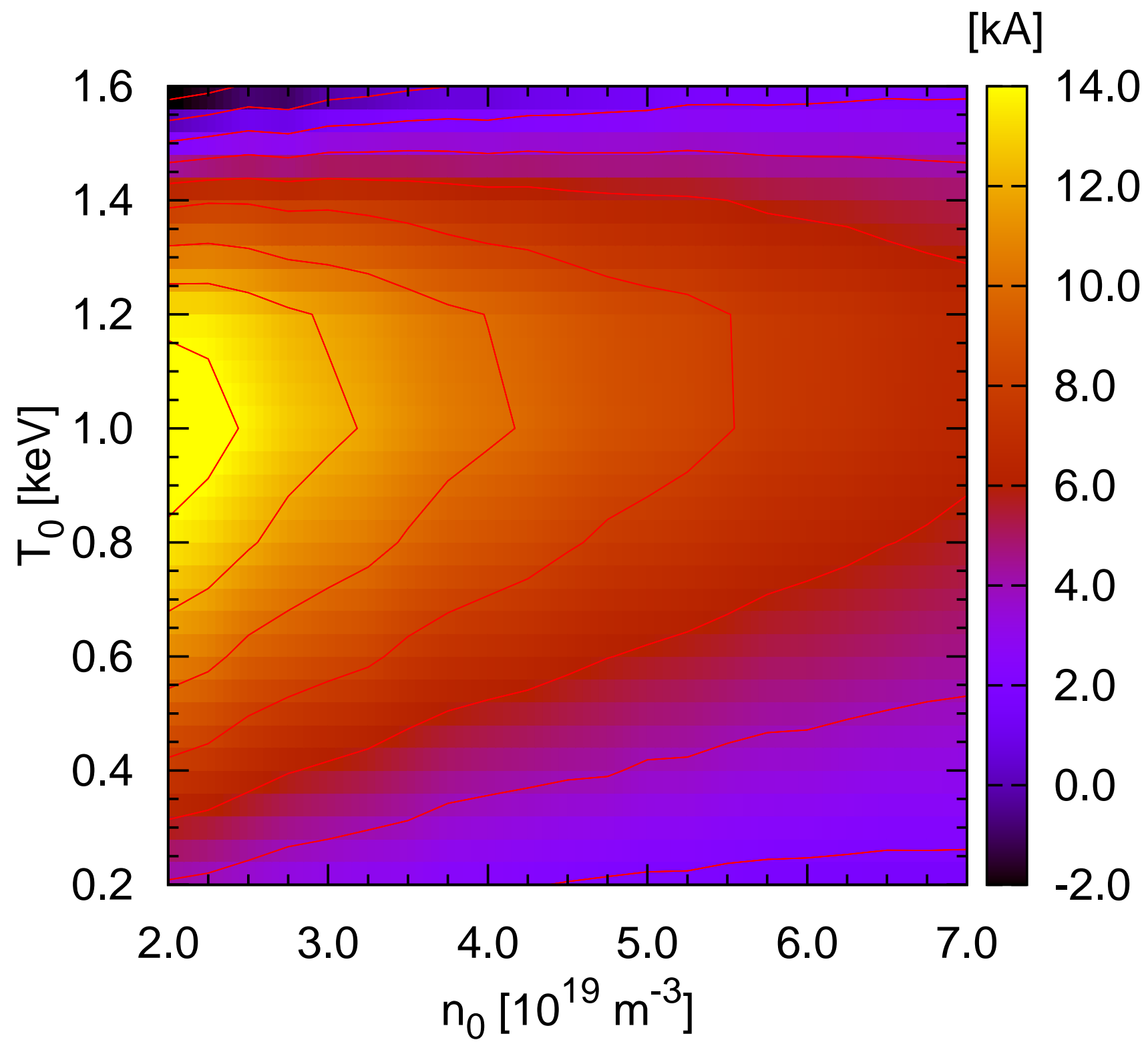

Figure 7. Total toroidal current $\left(I_{\mathrm{CD}}\right)$ obtained with the Lin-Liu model as a function of the central electron temperature $\left(T_{0}\right)$ and density $\left(n_{0}\right)$, keeping the analytical profiles as $n_{e}(\psi)=n_{0}\left(1-\psi^{2}\right)^{7}$ and $T_{e}(\psi)=T_{0}\left(1-\psi^{7}\right)^{10}$, with $\psi=\rho^{2}$ the magnetic flux 


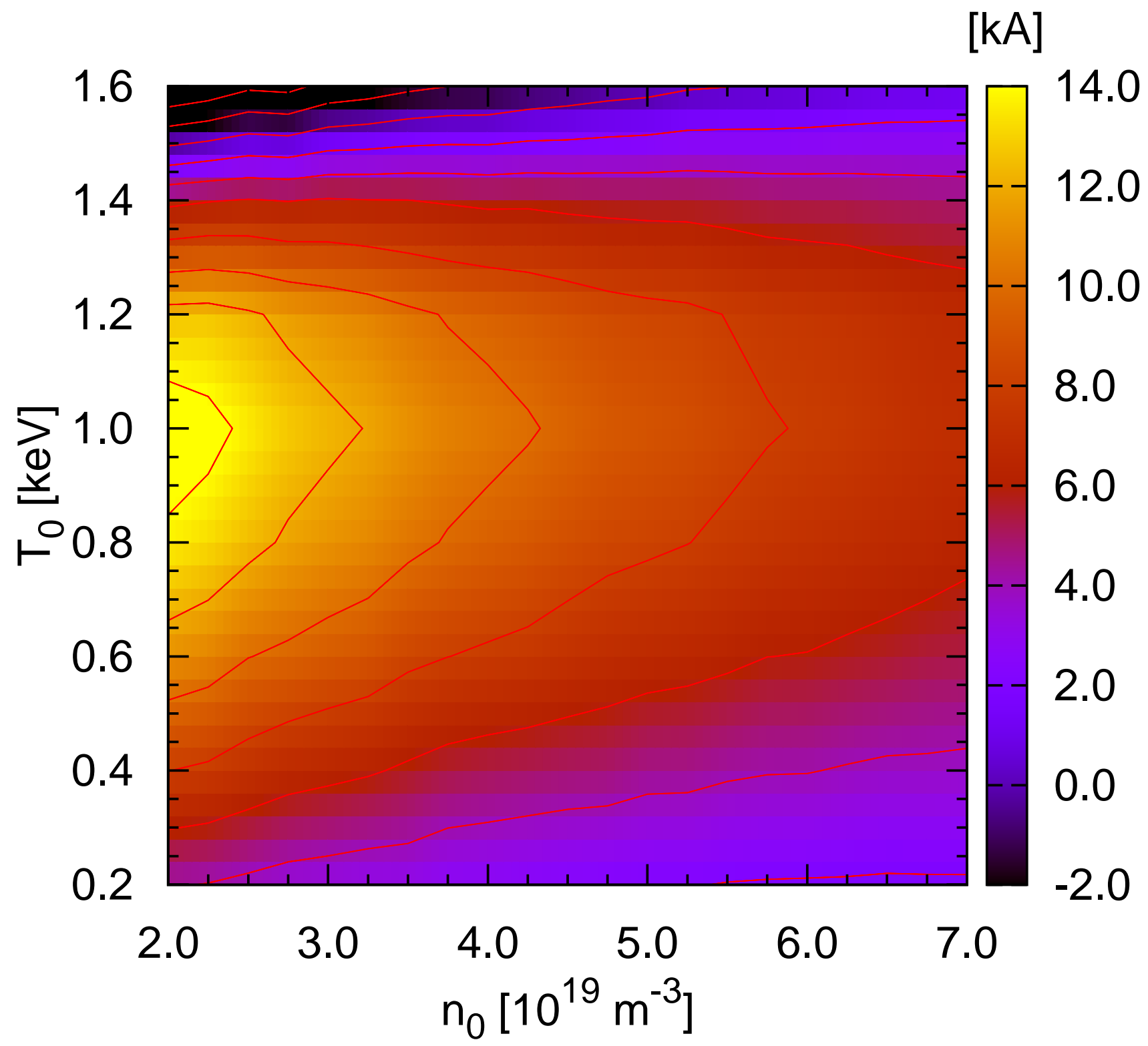

Figure 8. Total toroidal current $I_{\mathrm{CD}}$ obtained with the mc model as a function of the central electron temperature $\left(T_{0}\right)$ and density $\left(n_{0}\right)$, and same profiles as in figure 7 . 


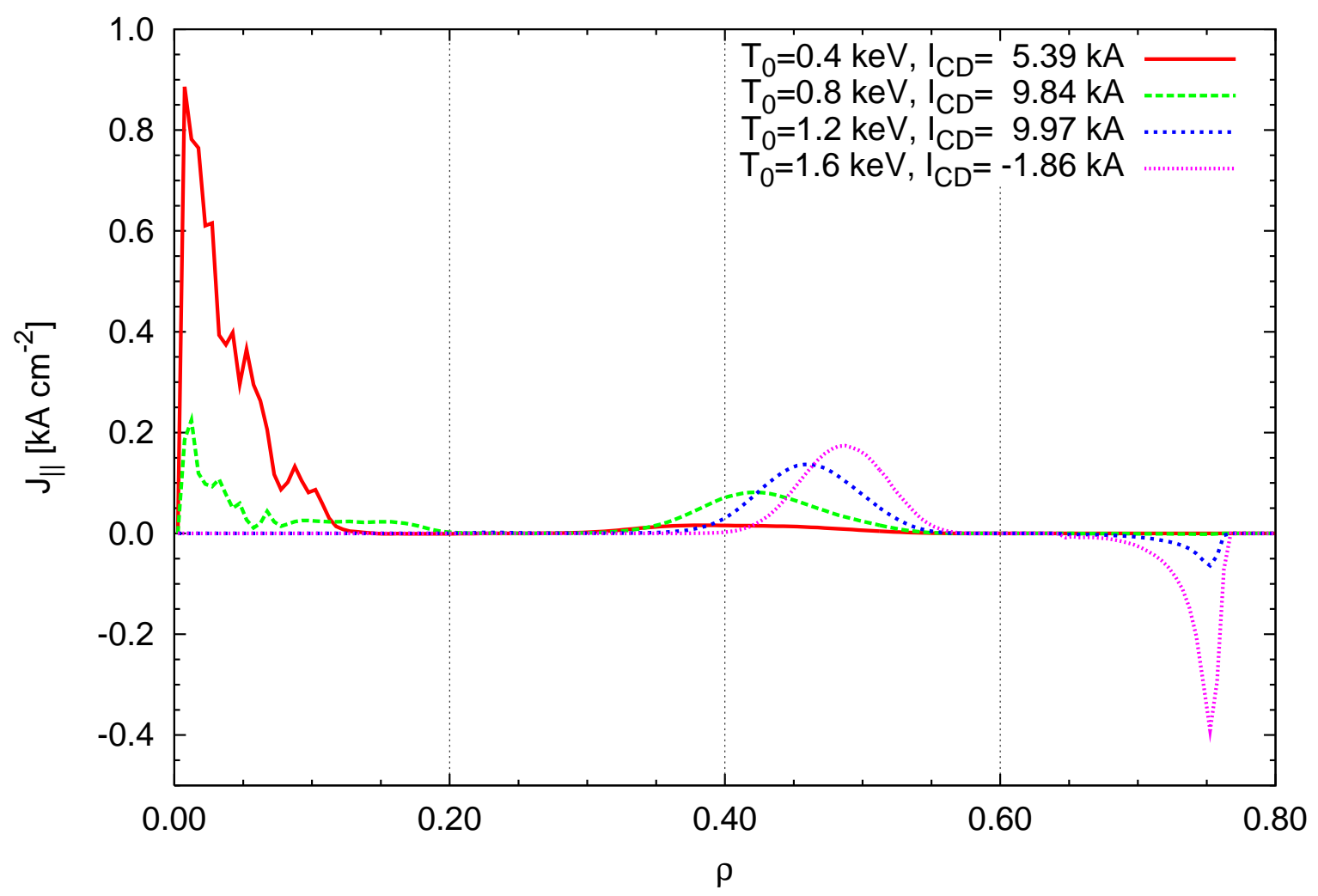

Figure 9. Current density radial profiles, $J_{\|}(\rho)$, for different values of $T_{0}$, and constant central density of $n_{0}=4.0 \times 10^{-19} \mathrm{~m}^{-3}$. The weakly relativistic mc model is used. 

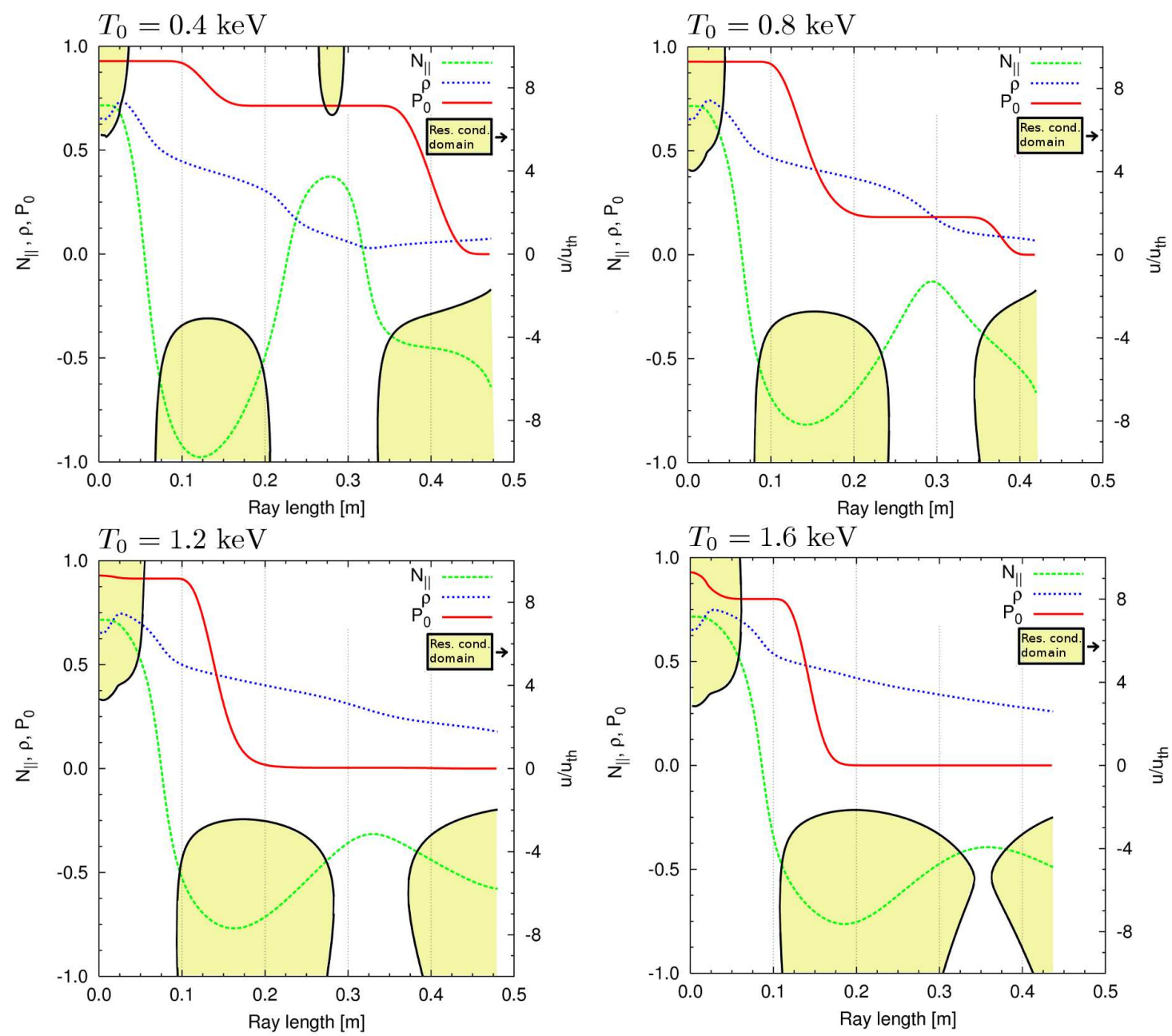

Figure 10. Referred to the left y-axis, $N_{\|}$(dashed line), effective radius $\rho$ (dotted line) and ray normalized power (solid line), as function of the length travelled by the central transmitted ray of the simulations that provide the current density profiles represented in figure 9. Same central temperature values than in figure 9 are considered, 0.4, 0.8, 1.2 and $1.6 \mathrm{keV}$. The power carried by the transmitted ray is normalized to the launched power, thus the initial value corresponds to the O-X conversion efficiency of the ray. The resonance condition domain is represented by the delimited coloured areas and are referred to the right y-axis. 


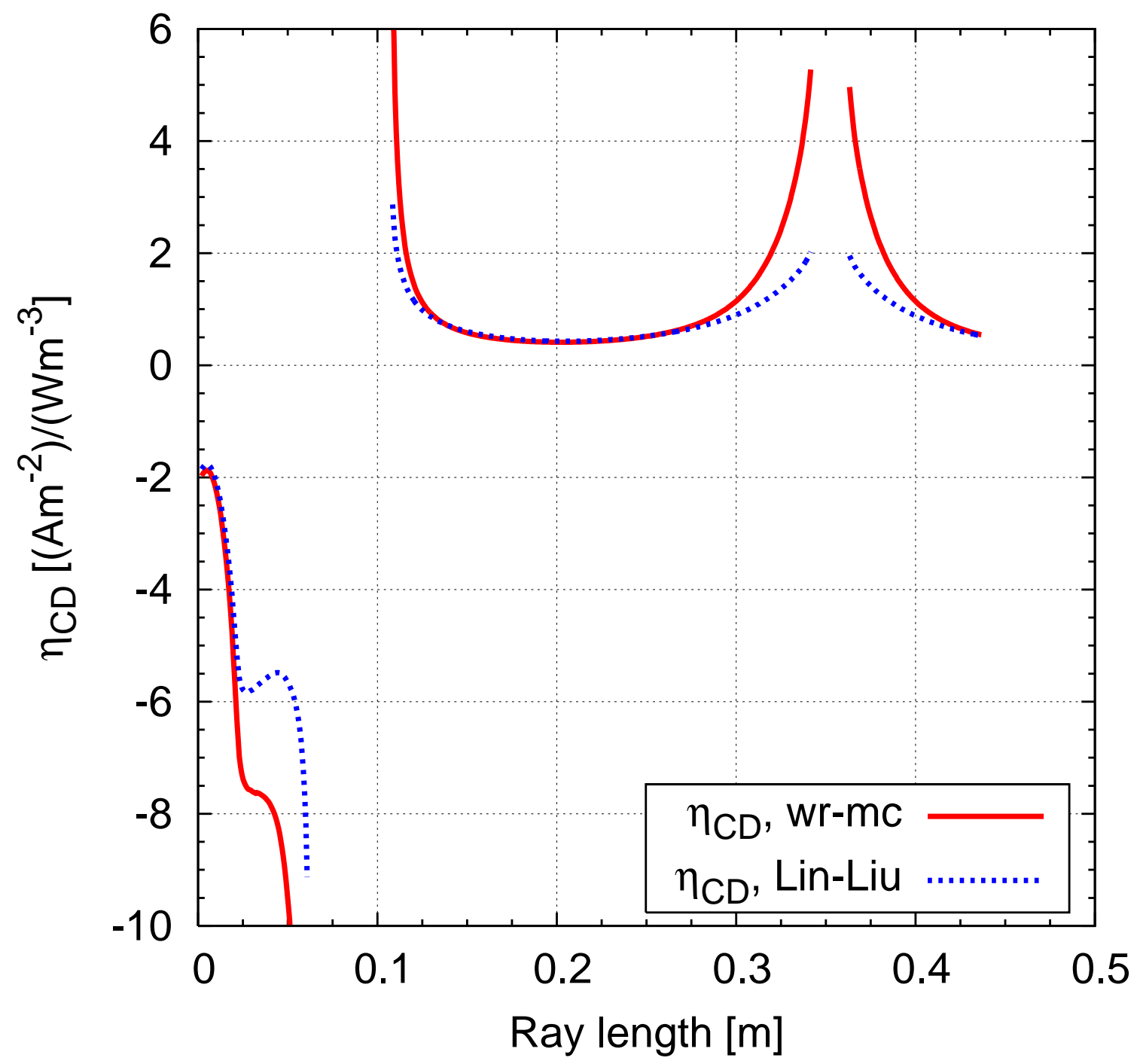

Figure 11. Current drive efficiency $\eta_{\mathrm{CD}}$ as a function of the transmitted ray length for the weakly relativistic mc and Lin-Liu models (solid and dotted lines respectively), and for the central ray of the multi-ray simulation used for the case with $T_{0}=1.6 \mathrm{keV}$ in figures 9 and 10. 


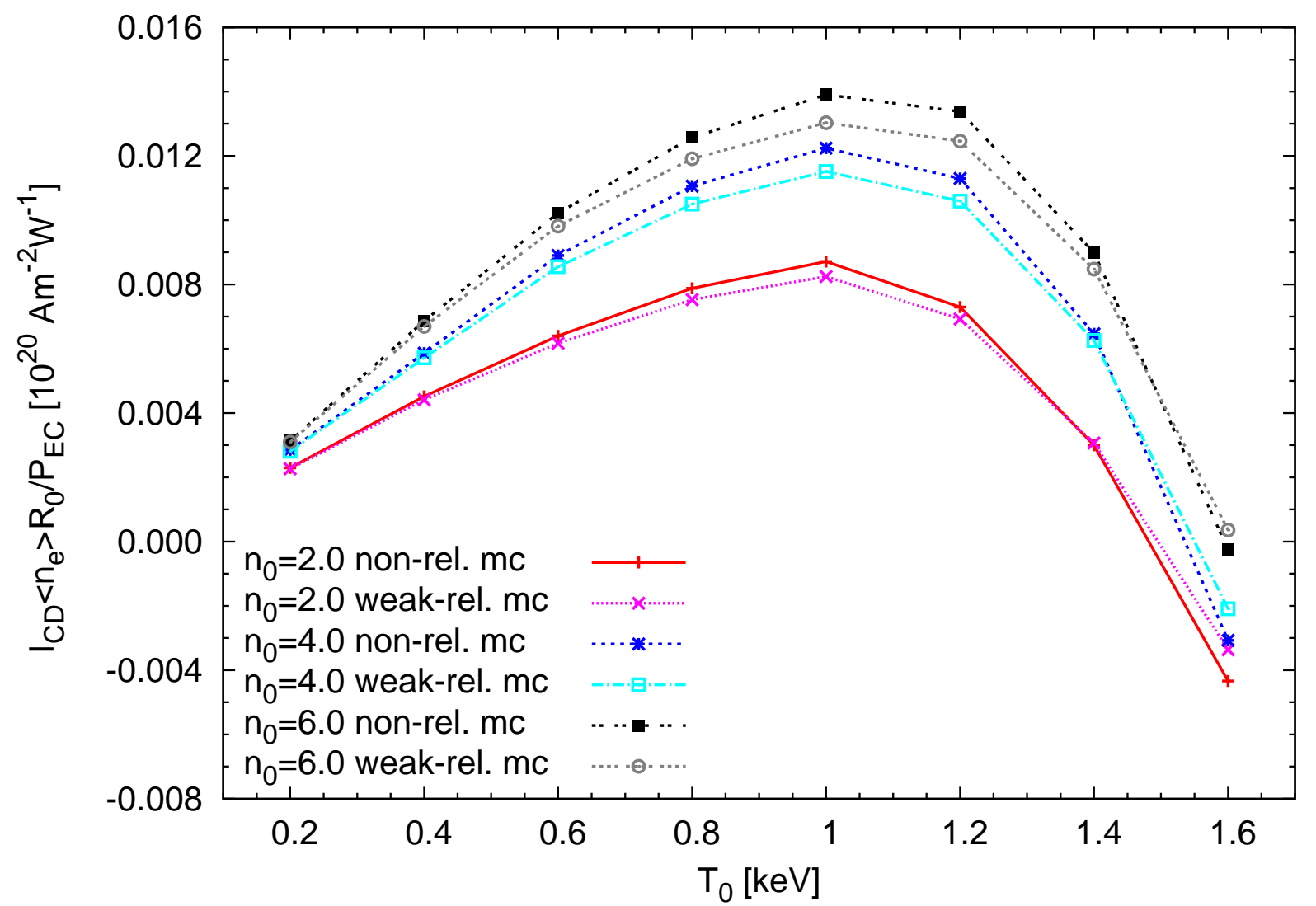

Figure 12. Total current drive efficiency, $I_{\mathrm{CD}}\left\langle n_{e}\right\rangle R_{0} / P_{\mathrm{EC}}$, as a function of the central temperature, for different central densities, and comparing the results under the weakly relativistic and non relativistic models 


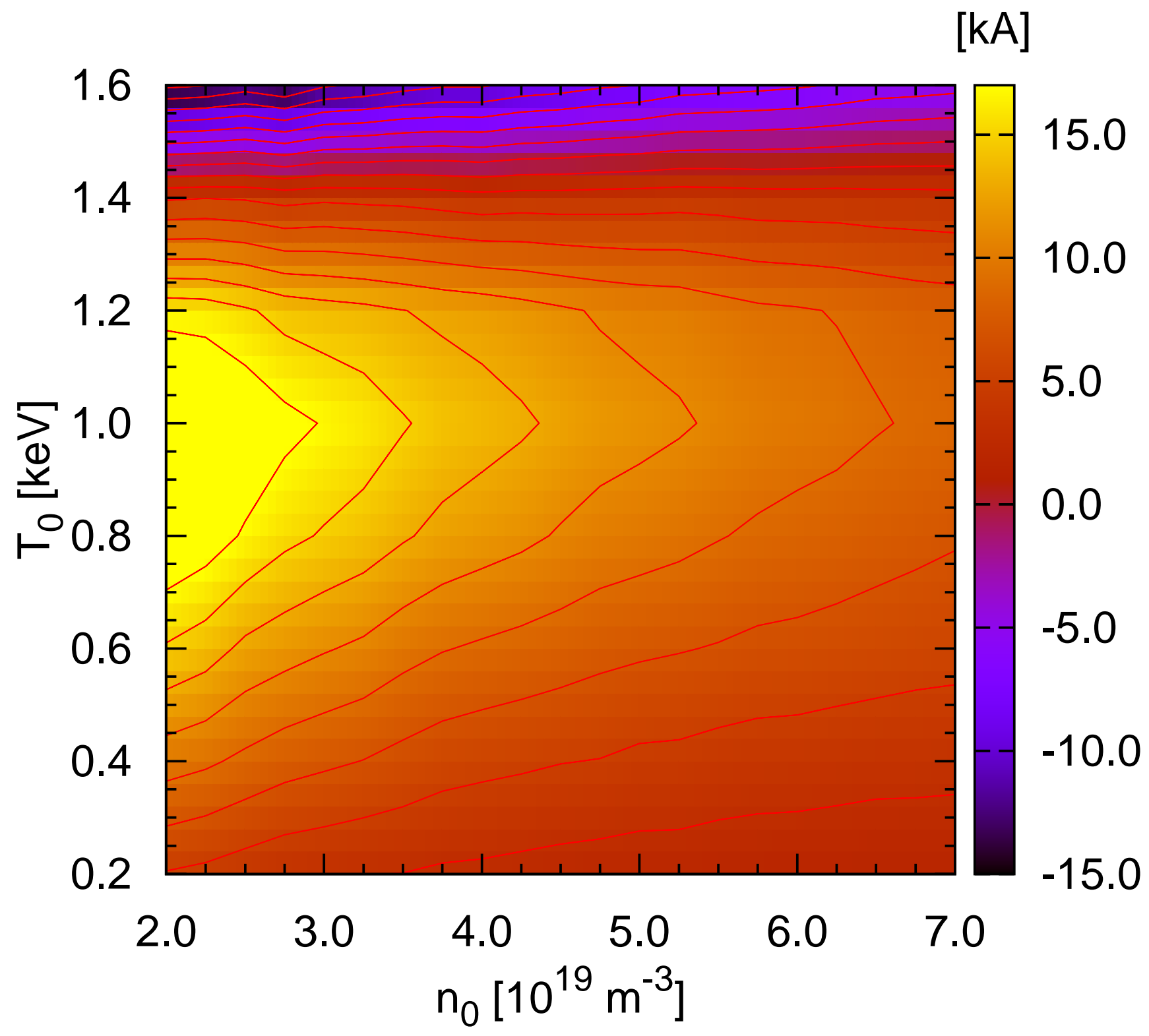

Figure 13. Total toroidal current $\left(I_{\mathrm{CD}}\right)$ obtained with the Ohkawa-corrected Fisch model as a function of the central electron temperature $\left(T_{0}\right)$ and density $\left(n_{0}\right)$, and same profiles as in figure 7 and 8 . 


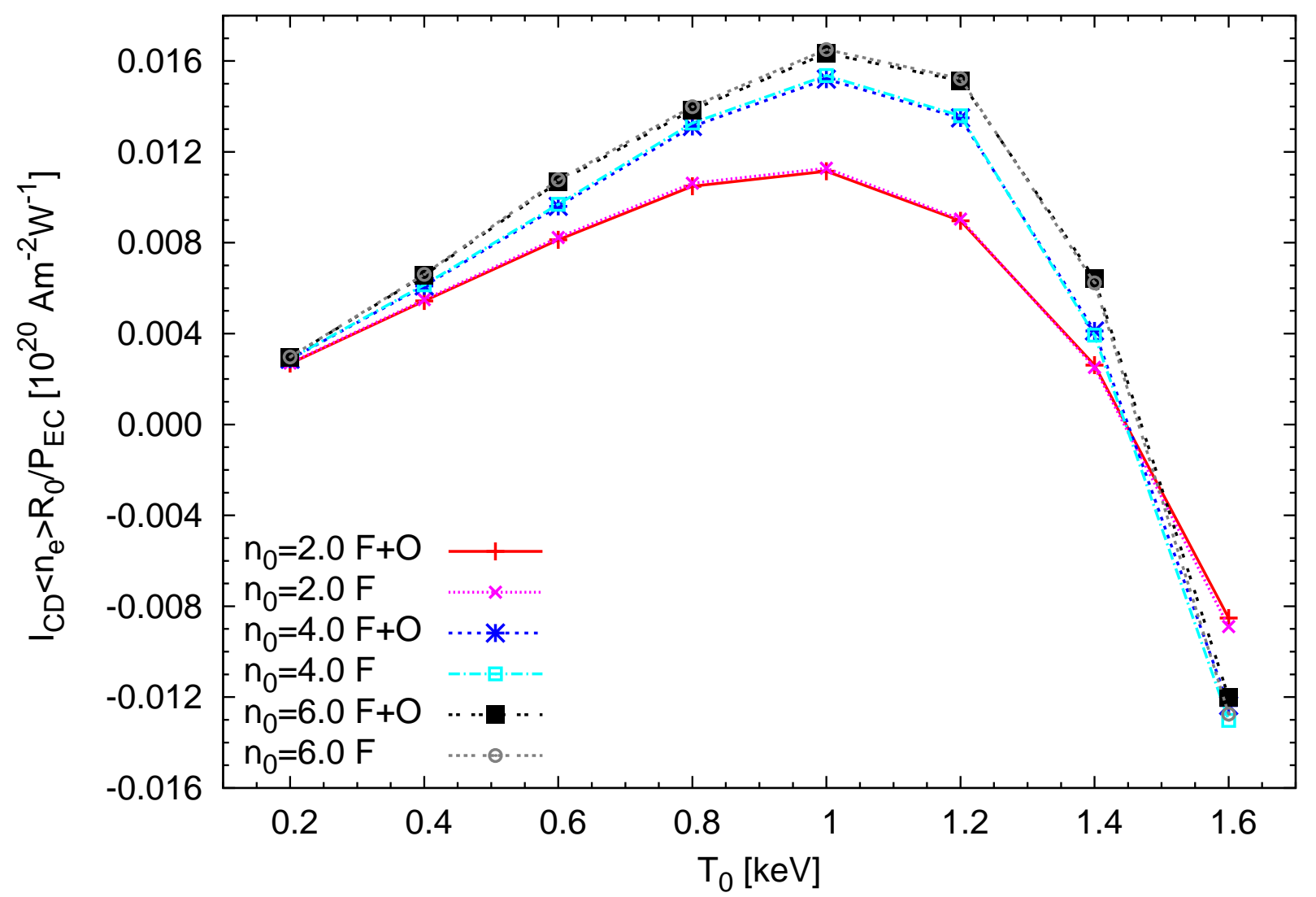

Figure 14. Total current drive efficiency, $I_{\mathrm{CD}}\left\langle n_{e}\right\rangle R_{0} / P_{\mathrm{EC}}$, as a function of the central temperature, for different central densities, and comparing the results using the Fisch model with and without the Ohkawa effect 


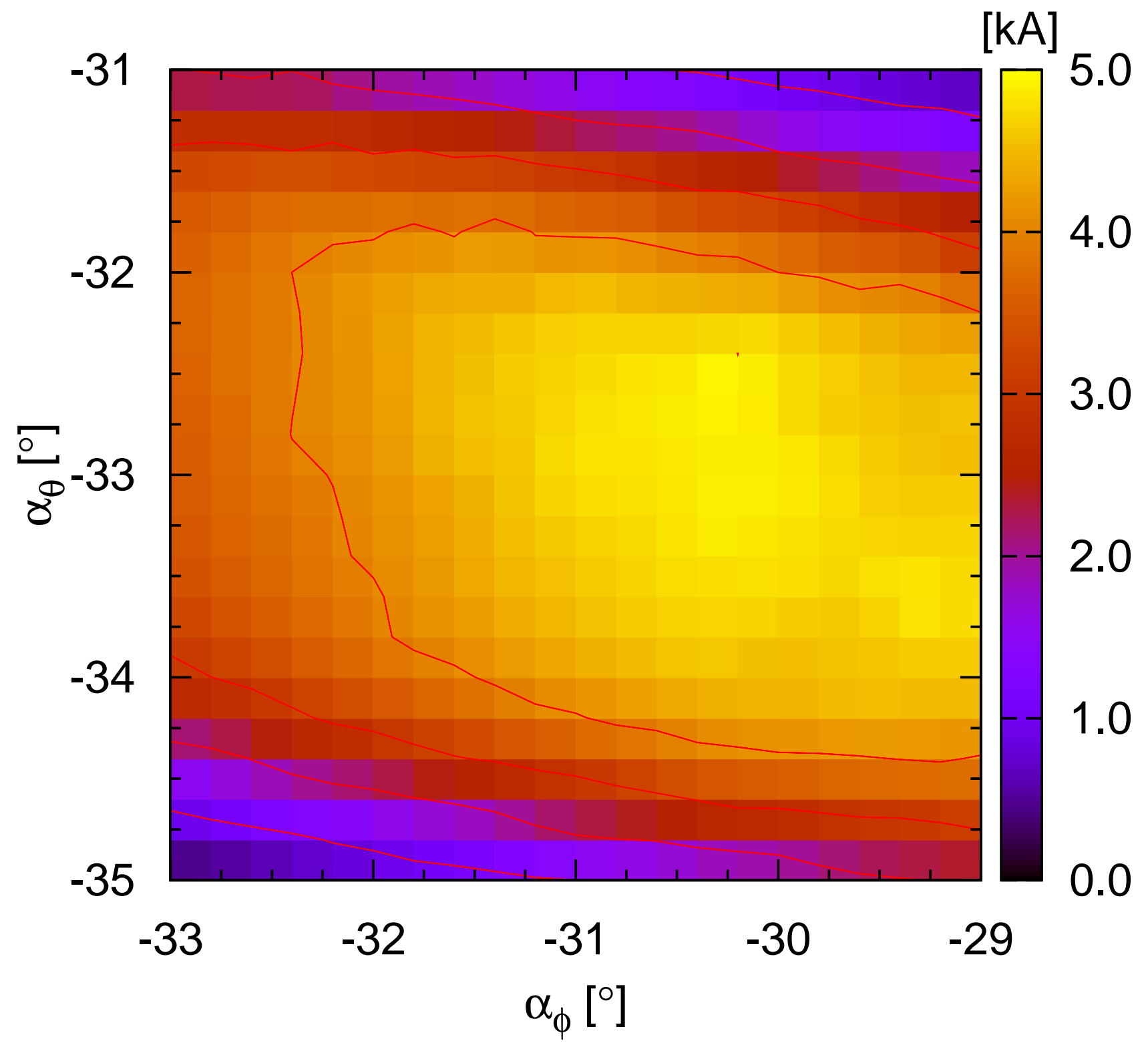

Figure 15. Total toroidal current $\left(I_{\mathrm{CD}}\right)$ obtained with the weakly relativistic mc model as a function of the position of the EBW heating system mirror, determined by the angles $\alpha_{\theta}$ and $\alpha_{\phi}$ 


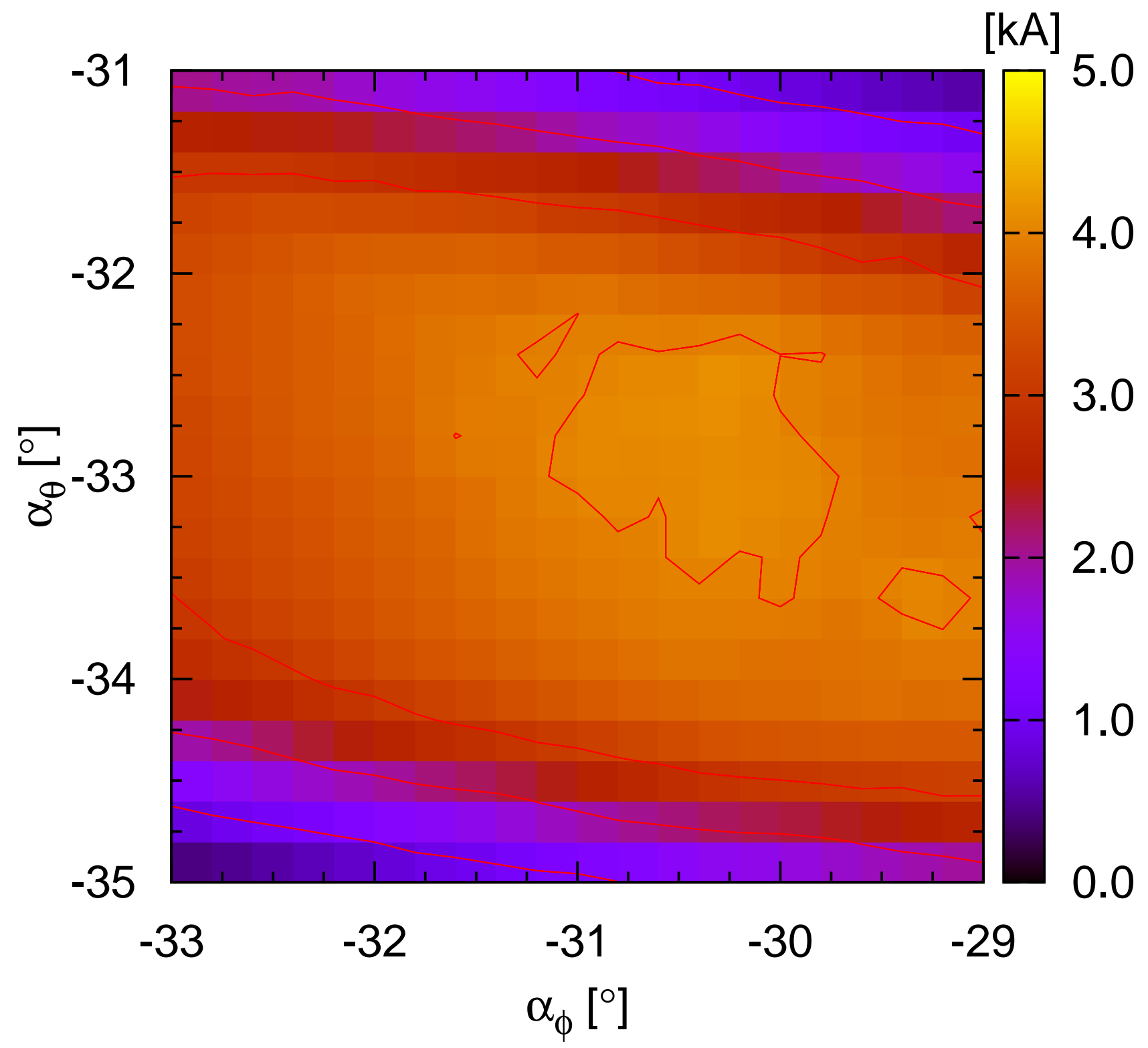

Figure 16. Total toroidal current $\left(I_{\mathrm{CD}}\right)$ obtained with the Lin-Liu model as a function of the the position of the EBW heating system mirror, determined by the angles $\alpha_{\theta}$ and $\alpha_{\phi}$ 


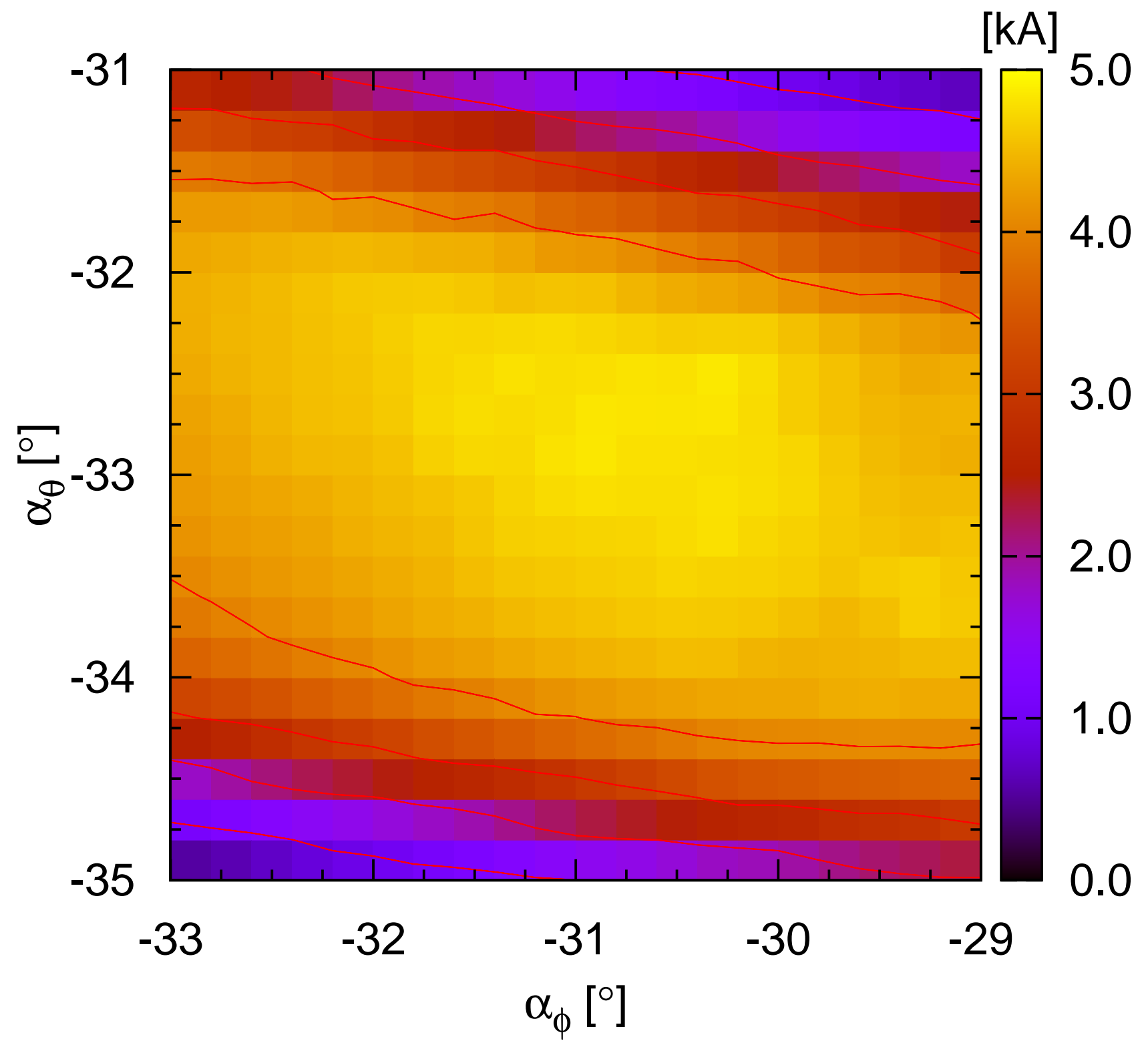

Figure 17. Total toroidal current $\left(I_{\mathrm{CD}}\right)$ obtained with the Ohkawa-corrected Fisch model as a function of the the position of the EBW heating system mirror, determined by the angles $\alpha_{\theta}$ and $\alpha_{\phi}$ 